\title{
Study on Arrival Aircraft Sequencing Based on Optimization of Point Merge Procedure
}

\author{
Yong Tian (D), Can Xu (D), Mengyuan Sun (D), Chao Li (iD, and Ruofei Sun (iD \\ College of Civil Aviation, Nanjing University of Aeronautics and Astronautics, Nanjing 210016, China \\ Correspondence should be addressed to Can Xu; xucan@nuaa.edu.cn
}

Received 18 October 2020; Revised 18 March 2021; Accepted 15 April 2021; Published 23 April 2021

Academic Editor: Junqing Li

Copyright (c) 2021 Yong Tian et al. This is an open access article distributed under the Creative Commons Attribution License, which permits unrestricted use, distribution, and reproduction in any medium, provided the original work is properly cited.

\begin{abstract}
With the air transport industry developing dramatically, the problem of flight congestion in the terminal area (TMA) has been aggravated. Particularly for arrival aircraft, the extraholding and delay not only increase the workload of air traffic controllers and aircrew but also reduce the operational efficiency. To struggle with this issue, it is crucial to study how to improve the operational efficiency of arrival aircraft. Under the background of the rapid promotion of Point Merge (PM) procedure program in the busy TMAs in the world, this paper firstly combines the optimization of PM procedure and arrival aircraft sequencing, aiming to maximize the operational benefits of arrival aircraft in TMA. Firstly, the framework of the PM procedure is set up to replace the traditional approach route known as Standard Instrument Arrival (STAR). After that, the PM procedure optimization model is established to provide a better approach route for each arrival aircraft. Then for a flow of arrival traffic, an aircraft sequencing model is established to readjust the landing sequence of arrival aircraft, thus further improving the operational benefits of arrival aircraft. Finally, taking the TMA of Shanghai Hongqiao International Airport as the case, we compare the impact of different flight procedures and different landing sequences on the operational benefits of arrival aircraft. The experimental results show that the application of the PM procedure optimization model and sequencing model can effectively improve the operational benefits on the premise of ensuring safety, thus realizing the safe and orderly approach in TMA.
\end{abstract}

\section{Introduction}

From 2015 to 2019, the civil aviation industry in China has witnessed rapid development, the total turnover of transportation has been increasing, and the annual growth rate has been kept above 5\% [1].

However, the rapid development of the air transport industry has caused a series of impacts on TMA. As an important area for aircraft taking off and landing, TMA is a very complex environment for flight operation. In TMA, the departure aircraft generally climb rapidly and join the airway after taking off with fewer conflicts and clearance, yet delays in arrival aircraft are common. The control for arrival aircraft is mainly based on STAR and radar vector. STAR which has a fixed route structure limits the flexibility of air traffic control and restricts the operational benefits of aircraft [2]. Compared with STAR, radar vector can make controllers control aircraft according to their expectations, which may increase the flexibility of control. However, with the soaring of traffic, radar vector leads to heavier workload of controllers due to more scattered trajectory, which may threaten the safety of flight [3]. Therefore, how to strengthen the flight management in TMA and carry out a safe and effective approach has become a key problem to realize TrajectoryBased Operation (TBO) in future civil aviation [4].

Combined with the full implementation of Area Navigation (RNAV) and Continuous Descent Approach (CDA) [5] technologies, the PM procedure is a new procedure which can enhance the predictability of flight trajectory and increase the capacity of TMA, therefore it is an effective exploration and innovation of TBO.

In 2006, the operational concept of PM was developed by the Norwegian Air Navigation Provider (AVNOR) in Oslo Advanced Sectorization and Automation Project (Oslo ASAP). In 2009, it was tested and used for the first time in the TMA of Oslo Airport in Norway. After that, it was 
successfully applied to the TMA of Dublin Airport in Ireland in 2012 and to the Area Control Center (ACC) of Paris in France in 2013. Then, PM technology has been highly valued by the Air Traffic Control (ATC) agencies of all European countries and has spread to Asia. On December 5, 2019, Shanghai Pudong International Airport officially tested the PM procedure, which marked the beginning of PM procedure application in China. According to statistics from Eurocontrol, there are more than 25 airports in 17 countries which have applied the PM procedure to date.

The successful trial operation of the PM procedure and its operational benefits have attracted a large number of scholars to conduct research on it. Some early studies focused on the structure of the PM procedure, aiming at exploring the adaptability of the PM procedure. Ivanescu et al. first proposed a vectorized model based on real-time simulation trajectory and developed a PM procedure that converged arrival flows on one single runway [6]. Subsequently, Favennec et al. showed how PM procedure can be applied to a typical TMA at the 9th Aviation Technology, Integration and Operations Conference [7]. Based on the operating environment of Istanbul Ataturk International Airport, Meric et al. proposed a PM procedure suitable for airspace designers and air navigation service providers [8]. After that, Chao et al. selected Runway 36 of Changsha Huanghua International Airport to design a PM procedure [9]. Considering the structure of the PM procedure, Zhao et al. further constructed a flight protection area and propose an obstacle assessment method for the PM procedure [10]. In the same year, Qi et al. analysed the advantages of the PM procedure in terms of communication, navigation, and surveillance [11]. In 2018, Sahin et al. evaluated the operational benefits of PM procedure in metroplex airports [12].

With more studies being carried out, some scholars began to study the new structure of the PM procedure. Liang et al. considered the operating environment of the TMA of Beijing Capital International Airport and adopted a new structure of PM procedure shaped like " $\infty$ " [13]. After that, they proposed a new multilayer PM path structure, which allocates the corresponding flight level to different types of aircraft lying on the same sequencing leg [14]. And in subsequent studies, the structure of the PM procedure was applied to build a complete approach and departure framework [15]. However, most PM procedure in the above studies are derived from the subjective design of researchers, which may not be the optimal structure of PM procedure. To struggle with this issue, Tian et al. iterated the key elements of the PM procedure to get the optimal structure of the PM procedure through the Genetic Algorithm (GA) [16].

In addition to the structure of the PM procedure, more and more scholars paid attention to the characteristics of traffic flow in the PM procedure, especially the sequence of arrival traffic. In 2016, Liang et al. simulated traffic conditions in PM procedure through RHC-SA hybrid algorithm and adopted $\mathrm{N}$-step-ahead to dynamically optimize the arrival traffic flow [17]. In the same year, Chen carried out the maximum flow analysis of the PM procedure with the method of network graph theory [18]. In 2017, considering the operational mode of aircraft in PM procedure, Wang et al. constructed a four-dimensional flight track prediction model [19]. In 2017, Hong et al. proposed an optimal and practical sequencing algorithm to increase the capacity in handling arrival aircraft through PM procedure [20]. In the same year, considering the uncertainty of the system, they improved the algorithm [21]. On this basis, they used auxiliary variables to transform the nonlinear equation into a linear equation to find the optimal solution through the Mixed Integer Linear Programming (MILP) algorithm [22]. Furthermore, an optimal sequencing algorithm of the PM procedure considering a holding pattern was proposed [23]. In 2020, Zhang et al. adopted a new metaheuristic algorithm to study arrival aircraft sequencing problem in PM procedure [24].

Generally speaking, the above studies demonstrated that the PM procedure can enlarge the capacity of TMA airspace and improve the economic benefit compared with the traditional STAR. However, most studies fail to combine the PM procedure with the readjustment of the sequence of arrival traffic in the PM procedure, as a result, the potential benefits of PM procedure cannot be fully realized. Besides, most studies only focused on the economic benefits of the PM procedure and the environmental benefits are ignored. Therefore, this paper combines the optimization of PM procedure and arrival aircraft sequencing, aiming to maximize the operational benefits of arrival aircraft in TMA, thus promoting the application of PM procedure, which has great significance for the sustainable development of air transportation.

The remainder of this paper is organized as follows. Section 2 gives a brief introduction of the framework of PM procedure, including its basic structure and the operation of arrival aircraft in PM procedure. In Section 3, GA is used to iterate the key elements of the PM procedure, and the optimized PM procedure can be obtained. Based on the special structure of the PM procedure, Section 4 proposes a sequencing model for arrival aircraft. In Section 5, we selected the TMA of Shanghai Hongqiao International Airport as the case to prove the validity of the study. This paper ends with some discussion and conclusion.

\section{Framework of PM Procedure}

PM procedure has a special structure; thus, the arrival aircraft in PM procedure need to adapt to its special structure. In this section, we construct the framework of PM procedure, including its structure and operation mode of arrival aircraft, to lay the groundwork for the optimization of PM procedure and arrival aircraft sequencing.

2.1. Structure of PM Procedure. This paper mainly studies the application of the PM procedure in TMA, so it is necessary to model the airspace of TMA. The airspace of TMA belongs to category C and D airspace. As shown in Figure 1, the midpoint of the entrance of the airport runway is taken as the origin. If it is a multirunway airport, the midpoint of the entrance of the main runway is taken as the origin. Through 


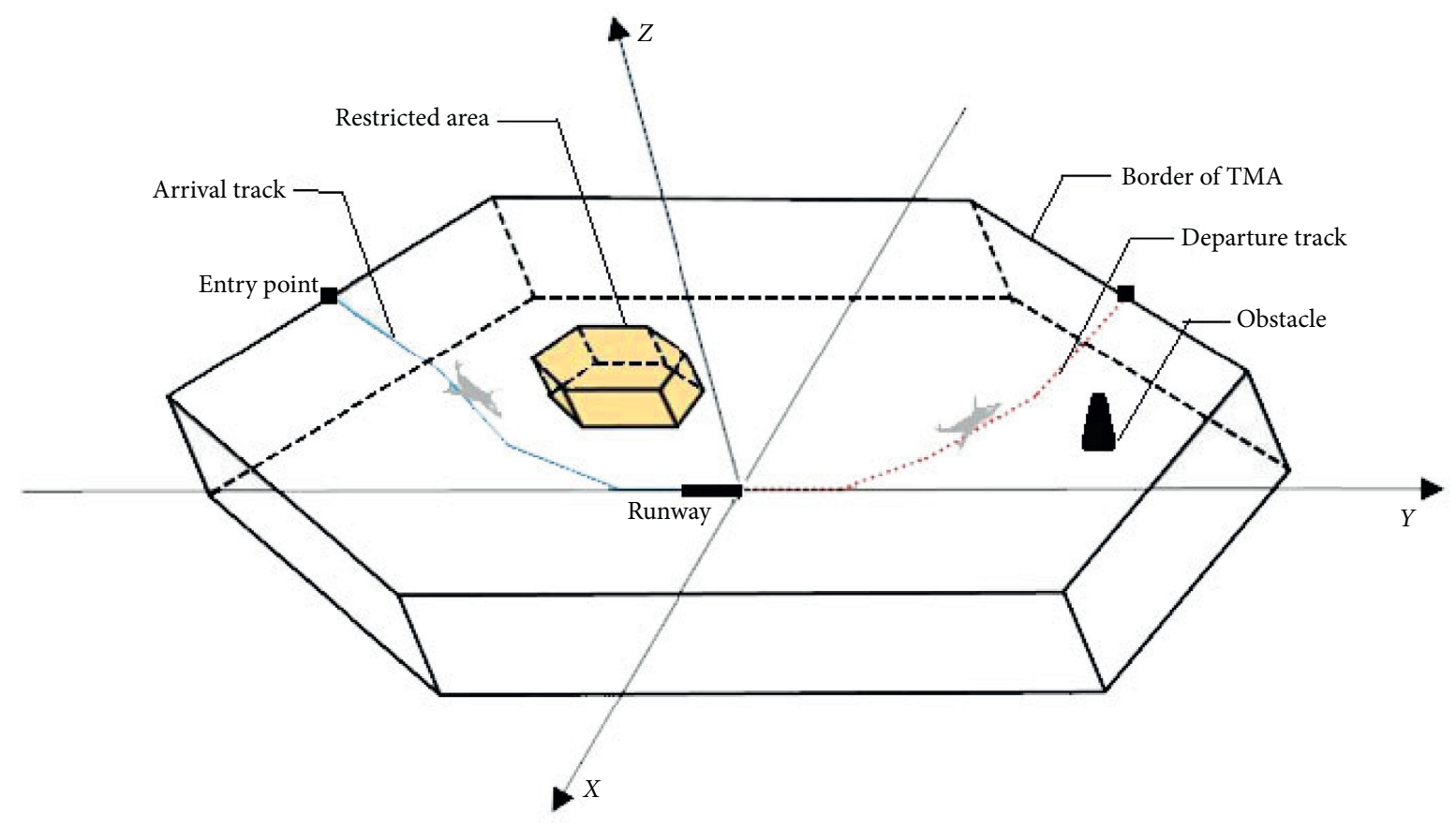

Figure 1: The model of TMA airspace.

the establishment of a Cartesian coordinate system, the air route, restricted area, and obstacle position distribution in TMA can be identified.

In TMA, as shown in Figure 2, a classic PM procedure for a single runway shaped like a fan includes merge point, sequencing legs, envelopes, and public segments. Among the elements that make up the PM procedure, the merge point and sequencing legs are the most critical.

Merge point is a fix in TMA, which is used to sort out and reorganize arrival traffic from different directions. Arrival aircraft fly past this point in an orderly manner according to the arrival sequence and follow a unified route to enter the intermediate or final approach. Sequencing legs are a series of predefined arcs to stretch or shorten the flight path for each arrival aircraft. The distance from any point on the sequencing leg to the merge point is equal. Besides, the waypoints at the end of each sequencing leg are the fly-over waypoints, and the other waypoints should be the fly-by waypoints. The controller can adjust the flight separation and sequence by controlling the moment when the aircraft turns to the merge point.

For safety, the sequencing legs shall be separated in at least one dimension: laterally or vertically. Consequently, the three options are as shown in Figure 3.

(1) Parallel legs with full overlap, as shown in Figure 3(a), with level off (constrained descent all along the legs may also be possible).

(2) Parallel legs with partial overlap, as shown in Figure 3(b), with constrained descent for the overlapping part (level off is also possible).

(3) Parallel legs with no overlap (dissociated), as shown in Figure 3(c), with an unconstrained descent (however a vertical separation may be required

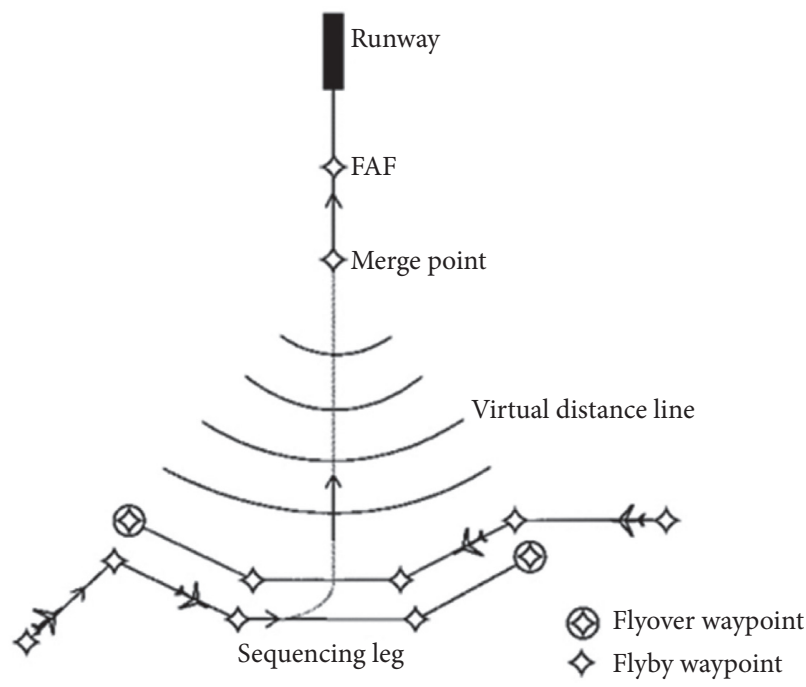

Figure 2: Classic PM procedure.

between the ends of leg run-off, subject to further safety assessment).

It is worth mentioning that there are some angle settings that need to be paid attention to during the generation of the PM procedure. The main angles include merge angle and offset angle. Merge angle is the apex of the sector and it can vary between $30^{\circ}$ and $120^{\circ}$. The offset angle is the angle between the bisector of merge angle and the extension of runway centerline. If offset angle equals 0 , the extension of the runway centerline just is the bisector of the merge angle. Commonly, offset angle can vary between $-90^{\circ}$ and $90^{\circ}$. When the bisector of merge angle is on the left of extension 


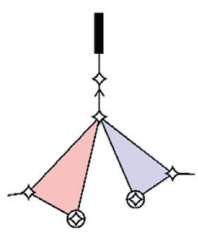

(a)

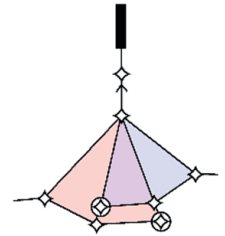

(b)

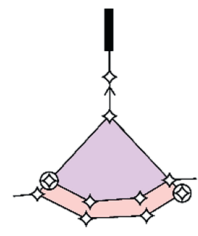

(c)

Figure 3: Three layout options of sequencing legs. (a) Parallel legs with full overlap. (b) Parallel legs with partial overlap. (c) Parallel legs with no overlap.

of the runway centerline, the offset angle is positive; otherwise, the offset angle is negative.

\subsection{Operation of Arrival Aircraft}

2.2.1. Kinematic Characteristics of Aircraft. In order to calculate the trajectory of arrival aircraft in the PM procedure, the kinematic characteristics of aircraft need to be considered. The motion of the aircraft can be described accurately by a six-degree-of-freedom model [25]. However, it requires extensive knowledge of aerodynamic models, propulsion models, and aircraft inertia tensor. Considering the complexity of the model, relatively simple basic kinematics models are used in the research of air traffic management fields. These kinematics models directly model the path characteristics of the aircraft without the need for a basic physical model. In 2007, Hull proposed an aircraft point mass model between the six-degree-of-freedom model and the basic kinematics model [26], which is a dynamics method considered to be accurate enough for aircraft trajectory calculation and simulation.

Assuming that all forces are applied to the gravity center of the aircraft, the motion of arrival aircraft will be reduced to three degrees of freedom. The forces on the aircraft are shown in Figure 4. We can use the following formula to calculate the lift $F_{L}$ and $\operatorname{drag} F_{D}$ :

$$
\begin{gathered}
F_{L}=\frac{C_{L} \rho V_{T}^{2} S}{2}, \\
F_{D}=\frac{C_{D} \rho V_{T}^{2} S}{2},
\end{gathered}
$$

where $C_{L}$ is the lift coefficient, $C_{D}$ is the drag coefficient, $\rho$ is the air density, $V_{T}$ is the true airspeed of the aircraft, and $S$ is the wing area.

In the point mass model, there is no need to model its inertia tensor or stability control, only aerodynamic, thrust, and gravity models are considered, and the total energy model of the aircraft can be derived as

$$
\left(F_{T}-F_{D}\right) \cdot V_{T}=m g \frac{\mathrm{d} H}{\mathrm{~d} t}+m V_{T} \frac{\mathrm{d} V_{T}}{\mathrm{~d} t}
$$

where $F_{T}$ is the total thrust, $F_{D}$ is the total drag, $m$ is the mass of arrival aircraft, $g$ is the acceleration of gravity, and $H$ is the flight altitude of arrival aircraft.

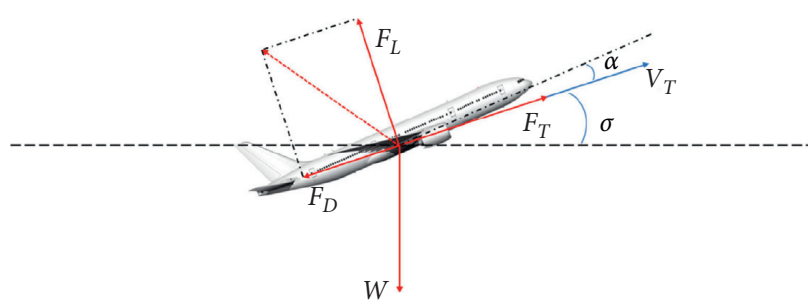

FIGURE 4: Forces on the aircraft.

When the flight trajectory calculation is involved in the experiment, we can obtain the thrust and speed information provided by Base of Aircraft Data (BADA) to calculate the position and status of the aircraft:

$$
\left\{\begin{array}{l}
\dot{x}=V_{T} \cos \sigma \sin \varepsilon, \\
\dot{y}=V_{T} \cos \sigma \cos \varepsilon, \\
\dot{z}=V_{T} \sin \sigma, \\
\dot{\varepsilon}=\frac{g \tan \mu}{V_{T}}, \\
\dot{H}=\frac{V_{T} \cdot\left(F_{T}-F_{D}\right)}{m g},
\end{array}\right.
$$

where $x, y, z$ are the coordinates of arrival aircraft, $\sigma$ is the flight track angle, $\varepsilon$ is the heading angle of arrival aircraft, and $\mu$ is the slope angle of arrival aircraft.

2.2.2. Conflict Detection and Resolution. For one aircraft operating in the PM procedure, the kinematic characteristics described in Section 2.2.1 can be used to calculate and simulate the flight track, while in the actual operation, when the arrival traffic flow contains more than one aircraft, we must consider the conflicts between aircraft and aircraft tracks to ensure the safety of operation. Therefore, we refer to the current risk assessment method of flight procedure and add new ideas based on the characteristics of PM procedure, thus realizing conflict detection and resolution for arrival traffic in PM procedure.

We divide collision risk into two categories: overtaking conflict (same path) and merging conflict (different path). 
Overtaking conflict refers to an accident in which the aircraft are located on the sequencing leg and the separation between two adjacent aircraft is gradually shortened due to the difference of speed. Merging conflict refers to the situation in which the distance between two adjacent aircraft gradually reduces when two aircraft are directed to the same merge point from different positions of the sequencing legs [27]. According to the flight operation process in the PM procedure, conflict detection is divided into three situations as shown in Figure 5.

(1) As shown in Figure 5(a), aircraft $i$ follows aircraft $j$ along the same sequencing leg at the same level, and the distance between them should meet International Civil Aviation Organization (ICAO) [28]-regulated wake turbulence minimum.

(2) As shown in Figure 5(b), aircraft $i$ and aircraft $j$ follow different sequencing legs with sufficient vertical separation. When aircraft $j$ turns to the merge point, $i$ shall continue to fly along the leg until the separation between two aircraft meets the ICAOregulated wake turbulence minimum.

(3) As shown in Figure 5(c), all aircraft in the procedure are at the same altitude when they arrive at the merge point. To eliminate the possibility of collisions caused by aircraft from different directions reaching the merge point, the wake turbulence separation is applied as a limit to control the distance between two adjacent aircraft. Besides, the use of the minimum wake turbulence separation is conducive to predict the Required Time of Arrival (RTA) of arrival aircraft, which can be combined with the Arrival Management (AMAN) system to better regulate the arrival flow.

To sum up, when arrival aircraft fly along the sequencing legs, we just need to check whether the distance between two adjacent aircraft on the same sequencing leg satisfies the distance-based minimum wake separation, thus realizing conflict detection. When arrival aircraft receives an instruction from the controller to fly directly to the merge point without continuing to fly along the sequencing leg, the RTA of each aircraft reaching merge point is calculated, and the RTA difference between two adjacent aircraft is compared to determine whether the time-based minimum wake separation is met, thus realizing conflict detection [13]. The ICAO-regulated wake turbulence minimum is shown in Table 1 [29].

Once a conflict is detected, we can control the time when the aircraft entries PM procedure, adjust the time when the aircraft can leave the sequencing leg, and limit the entry speed of the procedure to realize conflict resolution in PM procedure [27].

\section{PM Procedure Optimization}

The design of the PM procedure directly affects the operation safety and operational benefits of arrival aircraft. Since the relevant elements in the PM procedure introduced in Section
2 only follow the basic principles of design, different designers have different values for these elements, which has different impact on the operational benefits of arrival aircraft in the PM procedure. Therefore, this section establishes an optimization model of the PM procedure to obtain the optimal PM procedure with better operational benefits for arrival aircraft.

To standardize and simplify the optimization problem of PM procedure, the model is assumed as follows:

(1) Regardless of navigation deviation, aircraft may follow the route specified by the controller.

(2) The simulation space covers the entire TMA; all airspace in TMA can be used except special airspace, including prohibited area, restricted area, and waning area.

(3) There are no special hazardous conditions on the operation of arrival aircraft, such as bad weather and fuel starvation.

3.1. Objective Function. Current researches mainly analyse and evaluate flight procedure from the aspects of safety, economy, and environment. Safety is a necessary guarantee for civil aviation work. Therefore, we take safety indexes as constraints and set conflict number equal to 0 . With regard to the economic benefits, the average flight time $T_{A}$ and the fuel consumption $F_{A}$ of each aircraft are chosen as indexes. And for environmental benefits, noise impact range $Q(L)$ and average pollutant emissions $E_{A}$ are chosen as indexes [30]. The specific calculation formula of the four indexes can refer to [16]. It is worth mentioning that the pollutant emissions are greatly affected by meteorological conditions, which is neglected by [16]. Therefore, in order to make the calculation more accurate, meteorological data from TMA are introduced to modify the pollutant emissions that needed to be controlled in the Engine Emissions Data Bank (EEDB) published by ICAO. The calculation and revised method are as follows [30]:

$$
\begin{aligned}
& \left\{\begin{array}{l}
\mathrm{EI}_{\mathrm{NO}_{x}}=\mathrm{EI}_{\mathrm{NO}_{x}}^{R} \cdot e^{\mathrm{CH}} \cdot \sqrt{\frac{\tau^{1.02}}{\xi^{3.3}}}, \\
\mathrm{EI}_{\mathrm{HC}}=\mathrm{EI}_{\mathrm{HC}}^{R} \cdot \frac{\xi^{3.3}}{\tau^{1.02}}, \\
\mathrm{EI}_{\mathrm{CO}}=\mathrm{EI}_{\mathrm{CO}}^{R} \cdot \frac{\xi^{3.3}}{\tau^{1.02}},
\end{array}\right. \\
& \mathrm{CH}=-19 \cdot(\mathrm{HR}-0.00634) \text {, } \\
& \mathrm{HR}=\frac{\left(0.62197058 \cdot R_{H} \cdot P_{W}\right)}{P-\left(R_{H} \cdot P_{W}\right)}, \\
& P_{W}=611 \cdot 10^{\left(7.5 T_{0} /\left(273.3+T_{0}\right)\right)} \text {, } \\
& \xi=\frac{\left(273.15+T_{0}\right)}{288.15}, \\
& \tau=\frac{P}{101325}, \\
& F F=\frac{F F^{R} \cdot \tau}{\xi^{3.8} \cdot e^{0.2 \cdot M^{2}}},
\end{aligned}
$$




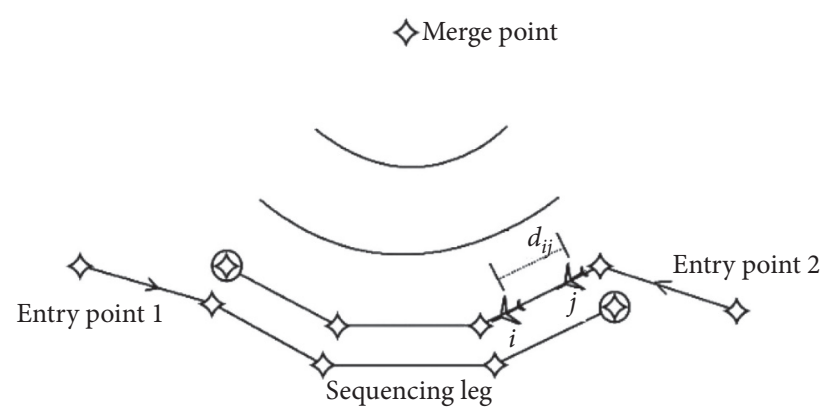

(a)

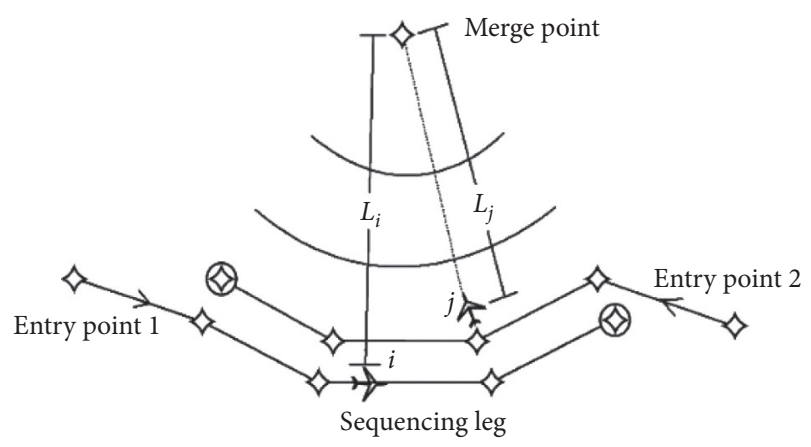

(b)

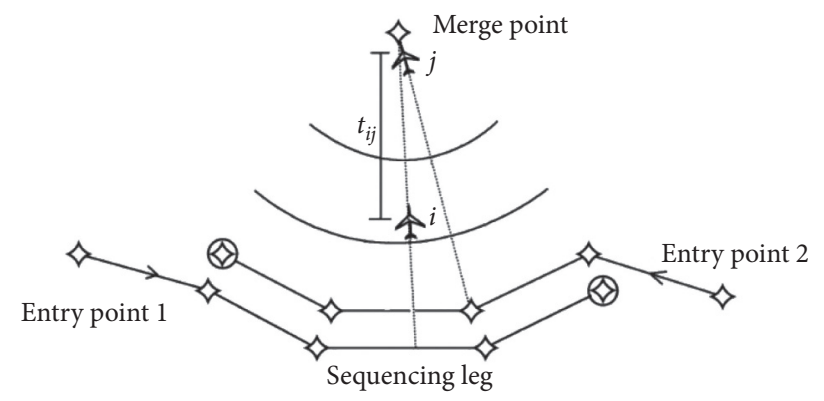

(c)

Figure 5: Three situations of conflict detection. (a) Entering. (b) Turning. (c) Merging.

TABLE 1: Aircraft minimum separation.

\begin{tabular}{lcccccc}
\hline & \multicolumn{5}{c}{ Trailing aircraft } \\
$\begin{array}{l}\text { Preceding } \\
\text { aircraft }\end{array}$ & $\begin{array}{c}\text { Distance-based } \\
\text { minimum separation } \\
\text { (nm) }\end{array}$ & $\begin{array}{c}\text { Time-based minimum } \\
\text { separation (s) }\end{array}$ \\
& Heavy & Medium & Light & Heavy & Medium & Light \\
\hline Heavy & 4 & 5 & 6 & 82 & 118 & 150 \\
Medium & 3 & 3 & 5 & 60 & 64 & 94 \\
Light & 3 & 3 & 5 & 60 & 64 & 68 \\
\hline
\end{tabular}

where $\mathrm{EI}_{\mathrm{NO}_{x}}, \mathrm{EI}_{\mathrm{HC}}$, and $\mathrm{EI}_{\mathrm{CO}}$ are the modified emission index of $\mathrm{NO}_{x}, \mathrm{CO}$, and $\mathrm{HC}$, respectively, $\mathrm{EI}_{\mathrm{NO}}^{R}, \mathrm{EI}_{\mathrm{HC}}^{R}$, and $\mathrm{EI}_{\mathrm{CO}}^{R}$ are reference emission indexes provided by $\mathrm{EDB}, \xi$ is the temperature ratio, $\tau$ is the pressure ratio, $R_{H}$ is the relative humidity, and HR is the humidity ratio, which reflects the amount of water in air per unit mass. $\mathrm{CH}$ is the humidity coefficient, $P_{W}$ is the saturation water vapor pressure, $T_{0}$ is the atmospheric temperature, $P$ is the atmospheric pressure, $\mathrm{FF}$ is the fuel flow rate, $\mathrm{FF}^{R}$ is the actual fuel flow rate, and $M$ is the Mach number.

As shown in Figure 6, the calculated fuel flow rate and emission index of CFM56-7B26s has a logarithmic regression relationship.

Based on the calculation methods of the four indexes, this paper adopts Analytic Hierarchy Process (AHP) to get their weight. The diagram of its tomographic structure is shown in Figure 7:

The following table is an example of the weights of each indicator obtained by 10 experienced controllers and 5

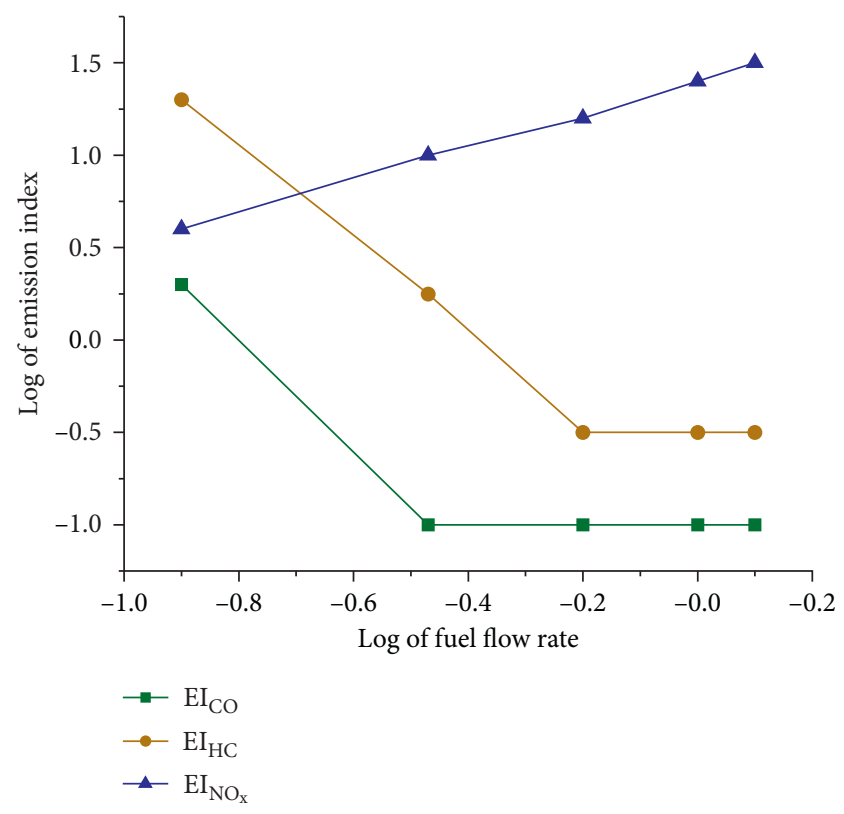

FIGURE 6: Logarithmic regression relationship between fuel flow rate and emission index.

professors. The experimental part of Section 5 adopts the weights shown in Table 2.

Finally, by the linear weighing-sum method, the multiobjective optimization is simplified to that of scalar objective optimization. The objective function is as follows:

$$
G=\min \left\{w_{1} T^{*}+w_{2} F^{*}+w_{3} E^{*}+w_{4} Q(L)^{*}\right\},
$$




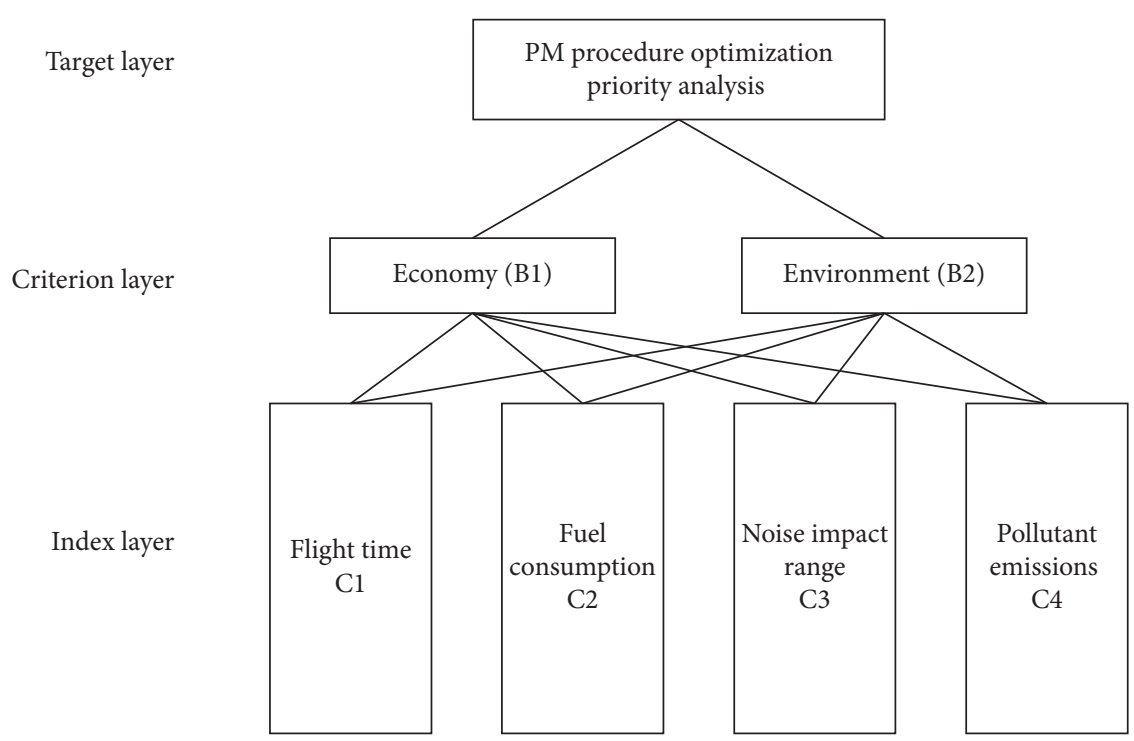

FIgURE 7: Tomographic structure model.

TABLE 2: The weight of each element.

\begin{tabular}{cccc}
\hline & $B_{1}$ & $B_{2}$ & Weight \\
\hline$C_{1}$ & 0.4287 & 0.1875 & 0.3759 \\
$C_{2}$ & 0.3573 & 0.125 & 0.3065 \\
$C_{3}$ & 0.1429 & 0.375 & 0.1937 \\
$C_{4}$ & 0.071 & 0.3125 & 0.1238 \\
\hline
\end{tabular}

where $w_{t}\left(\sum_{t=1}^{4} w_{t}=1\right)$ is the weighting factor of each index, which can be got by AHP mentioned above, and $T_{A}^{*}, F_{A}^{*}, E_{A}^{*}$, and $Q(L)^{*}$, respectively, denote $T_{A}, F_{A}, E_{A}$, and $Q(L)$ after being standardized by $\min -\max$ normalization.

3.2. Constraints. Considering the safety requirements of the flight procedure, we establish the following constraints to ensure that the optimized PM procedure can be applied in practice.

(1) Conflict-free constraint

$$
\begin{aligned}
& C=\sum_{i=1}^{n} c_{i}=0, \\
& c_{i}= \begin{cases}0, & d_{i j} \geq W_{i j}, \\
1, & d_{i j}<W_{i j},\end{cases}
\end{aligned}
$$

where $\mathrm{C}$ is the number of total conflicts, $c_{i}$ is the number of conflicts for aircraft $i, d_{i j}$ is the distance between aircraft $i$ and $j$, and $W_{i j}$ is ICAO-regulated wake turbulence minimum between aircraft $i$ and $j$.

(2) Clearance constraint

$$
H \geq H_{\text {OCA }}
$$

where $H$ is the altitude of airline and $H_{\mathrm{OCA}}$ is the obstacle clearance altitude which is the minimum safe altitude for the arrival aircraft.

(3) Other constraints

$$
\left\{\begin{array}{l}
30^{\circ} \leq \delta \leq 120^{\circ} \\
-90^{\circ} \leq \theta \leq 90^{\circ} \\
2000 \mathrm{ft} \leq H_{M} \leq 4000 \mathrm{ft} \\
8000 \mathrm{ft} \leq H_{S} \leq 12000 \mathrm{ft} \\
0 \leq \Delta \gamma_{i} \leq \delta
\end{array}\right.
$$

where $\delta$ is the merge angle, $\theta$ is the offset angle, $H_{M}$ and $H_{L}$, respectively, are the altitude of merge point and sequencing leg, and $\Delta \gamma_{i}$ is the angle of aircraft heading change when aircraft $i$ turns to the merge point.

3.3. Design of Optimization Algorithm. Due to the great difference in the magnitude of different indexes, we remove the units of the four indexes and transform them into dimensionless pure values by means of min-max normalization, so that indexes of different units or magnitudes can be compared and weighted. The replaced objective function can be expressed as

$$
\min g(\theta)=\sum_{j} w_{j} G_{j},
$$

where the weighting factor $w_{j}\left(\sum_{j} w_{j}=1\right)$ is the weight of each target.

In order to solve the above optimization model, the nonlinear least square estimation method based on GA is adopted. GA is a type of Evolutionary algorithm, which finds the optimal solution by imitating the mechanism of natural selection and genetics. It is a widely used algorithm with strong adaptability and robustness. In the algorithm, the population individual is denoted by the binary coding form of a multiparameter cascade. We use the code string-

$\mu_{1}, \mu_{2}, \ldots, \mu_{l 1} v_{1}, \nu_{2}, \ldots, v_{l 2} o_{1}, o_{2}, \ldots, o_{l 3} \kappa_{1}, \kappa_{2}, \ldots, \kappa_{l 4}$, of which length is $l_{1}, l_{2}, l_{3}$, and $l_{4}$, respectively, to $k_{k}$ denote the 
parameters to be evaluated. In the simulation process, we set the iteration number as 1000 , the crossover probability $P_{c}$ as 0.6 , the mutation probability $P_{m}$ as 0.6 , and the size of the population as 40 . We use binary encoding; the specific coding process in the algorithm is shown in Table 3.

\section{Arrival Aircraft Sequencing}

The optimized PM procedure can provide a better approach route for each arrival aircraft, thus improving the operational benefits of each aircraft. However, for the whole arrival traffic flow, the operational benefits still leave much space to improve. Therefore, in this section, the aircraft sequencing model in the PM procedure is constructed by using Constrained Position Shift (CPS) to provide a reference for controllers to make decisions in arrival aircraft sequencing, thus further improving the operational benefits of arrival traffic.

4.1. Problem Discrimination. Based on the characteristics of the aircraft sequencing problem, we imply the idea of graph theory to transform the sequencing problem, taking the aircraft as the node in the directed graph and the minimum safety separation required between two aircraft as the distance of the directed path in the graph. Through graph theory, the aircraft sequencing problem is equivalent to Traveling Salesman Problem (TSP) with asymmetric distance to solve the optimal Hamilton loop [31].

However, through solving the TSP problem, the aircraft sequence may change greatly, which leads to the aircraft land much earlier or later than scheduled. In consideration of the above problem, the application of CPS strategy can to some extent improve this situation, making the aircraft sequence readjustment more optimized and reasonable. Based on the CPS strategy, we establish a time window for advanced and delayed aircraft to restrict the earliest or latest landing time of each aircraft on account of aircraft performance.

4.2. Objective Function. Considering the role of PM procedure in improving economic and environmental benefits, typical indexes of these two aspects still were selected to adjust the landing sequence, namely, flight time and fuel consumption representing economic benefits, noise impact range, and pollutant emissions representing environmental benefits, while fuel consumption and emissions change mainly associated with the flight time. The calculation of noise is mainly associated with the flight procedure; adjustment of aircraft landing sequence has little effect on the low altitude trajectory of aircraft; thus, the noise changes little. Therefore, fuel consumption, pollutant emissions, and noise have no need to be considered in this section. Based on this, we took the minimum flight time of aircraft in TMA as the optimization objective and established the objective function as follows:
TABle 3: Binary coding process.

\begin{tabular}{lccc}
\hline Gene & Range & Precision & Code string length \\
\hline$\mu$ & $(30,120)$ & 5 & 25 \\
$\nu$ & $(-90,90)$ & 5 & 26 \\
$o$ & $(600,1500)$ & 300 & 22 \\
$\kappa$ & $(2400,4200)$ & 300 & 23 \\
\hline
\end{tabular}

$$
\min Z=\sum_{i=1}^{n}\left(s_{i}-t_{0, i}\right), \quad i=1,2, \ldots, n,
$$

where $Z$ is the total flight time of all aircraft in TMA from arrival to landing, $s_{i}$ is the time of aircraft $i$ reaching the threshold of runway, $s_{i}=t_{0, i}+t_{1, i}+t_{2, i}+t_{3, i}+t_{4, i}, t_{0, i}$ is the time of aircraft $i$ entering TMA, $t_{1, i}$ is the required time of aircraft $i$ entering PM procedure, $t_{2, i}$ is the time of aircraft $i$ flying along the sequencing leg, and $t_{3, i}$ is the time interval between the aircraft $i$ leaving the sequencing leg and reaching the merge point. $t_{4, i}$ is the time of aircraft $i$ flying from the merge point to the threshold of the runway. Since the sequencing of arrival aircraft is less likely to change the value of $t_{1, i}, t_{3, i}$, and $t_{4, i}$, we can set them as fixed values, and the objective function can be simplified as

$$
\min Z^{\prime}=\sum_{i=1}^{n} t_{2, i}, \quad i=1,2, \ldots, n \text {. }
$$

Through the above analysis, as long as the time of all aircraft flying along the sequencing legs is minimized, the total flight time of all aircraft from entering the TMA to reaching the runway threshold can be guaranteed to be the smallest. Therefore, we only need to find the landing sequence that minimizes the time of all aircraft flying along the sequencing leg, thus improving the operational benefits of arrival aircraft.

4.3. Constraints. To meet the needs of actual operation, the constraint conditions are established as follows:

(1) Flight uniqueness constraint

$$
\sum_{j=1}^{n} x_{i j}=1, \quad i=1,2, \ldots, n,
$$

where $x_{i j}$ is 0 - 1 decision variable; if aircraft $j$ follows aircraft $i, x_{i j}=1$; otherwise, $x_{i j}=0$.

(2) Separation constraint

According to the method of conflict detection and resolution, constraints are established for entry point, turning point, and merge point to ensure that the separation between adjacent aircraft always meets the minimum wake separation regulated by ICAO. 


$$
\begin{aligned}
{\left[\left(t_{0, j}-t_{0, i}\right)+\left(t_{1, j}-t_{1, i}\right)\right] \cdot x_{i j} \cdot C_{i j} \geq T_{i j} i, } & j=1,2, \ldots, n, i \neq j, \\
{\left[\left(t_{0, j}-t_{0, i}\right)+\left(t_{1, j}-t_{1, i}\right)+\left(t_{2, j}-t_{2, i}\right)\right] \cdot x_{i j} \geq T_{i j} i, } & j=1,2, \ldots, n, i \neq j, \\
{\left[\left(t_{0, j}-t_{0, i}\right)+\left(t_{1, j}-t_{1, i}\right)+\left(t_{2, j}-t_{2, i}\right)+\left(t_{3, j}-t_{3, i}\right)\right] \cdot x_{i j} \geq T_{i j} i, } & j=1,2, \ldots, n i \neq j,
\end{aligned}
$$

where $t_{0, j}$ is the time of aircraft $j$ entering TMA, $t_{1, j}$ is the required time of aircraft $j$ entering PM procedure, and $C_{i j}$ is 0-1 decision variable; if aircraft $i$ and $j$ lie on the same sequencing leg, $C_{i j}=1$. Otherwise, $C_{i j}=0 . T_{i j}$ is the minimum time-based separation between aircraft $i$ and $j, T_{i j} \neq T_{j i} . t_{2, j}$ is the time of aircraft $j$ flying along the sequencing leg, and $t_{3, j}$ is the time interval between the aircraft $j$ leaving the sequencing leg and reaching the merge point.

(3) Time window constraint

In order to avoid large changes on the sequence of arrival aircraft after sequencing, resulting in the aircraft landing much earlier or later than scheduled, we establish a time window for each arrival aircraft as follows:

$$
0 \leq t_{i, 2} \leq \frac{L_{i}}{V_{i}}, \quad i=1,2, \ldots, n,
$$

where $L_{i}$ is the length of the sequencing leg which aircraft $i$ lie on and $V_{i}$ is the speed of aircraft $i$ flying along the sequencing leg by utilizing the maximum allowable time of an aircraft flying along the sequencing leg to restrict great changes of aircraft sequence.

4.4. Design of Optimization Algorithm. The simulated annealing (SA) algorithm is a stochastic global optimization algorithm based on Monte-Carlo iteration, which starts from the study of the solid annealing process in physics. Some scholars have also proposed new and effective algorithms recently, such as the Improved Artificial Bee Colony (IABC) algorithm in [32]. The IABC algorithm uses three innovative strategies, a hybrid initialization strategy, a welldesigned encoding repair strategy, and a variable length local search strategy, which greatly overcome the shortcomings of the classical $A B C$ algorithm, thus more applicable for the vehicle routing problem with time windows (VRPTW). Compared with the newly proposed algorithms, the SA algorithm has been proposed for a long time but is still widely used for its ability to overstep the local extremum and applicability to different kinds of problems with continuous or discrete space states. Therefore, in view of the convenient implementation as well as reliable results, this paper adopts the SA algorithm to solve the arrival aircraft sequencing problem. In the aircraft sequencing problem, it is assumed that each aircraft combination in the sequence corresponds to the solid cooling state in the annealing algorithm, and the length of flight time corresponds to the energy of that state. The algorithm is used to solve the shortest flight time of arrival aircraft and the sequence corresponding to the shortest flight time. The specific process is shown in Figure 8.

(1) Initialization. It includes determining the initial temperature $T_{1}$ at the beginning of the algorithm, generating the initial landing sequence $O$ of the arrival traffic flow, setting the termination temperature $T_{2}$, iteration times $L$, and temperature decay coefficient $\varphi$.

(2) Inner Loop for $k=1,2, \ldots, L$. Under the constraint condition, a disturbance is generated randomly, and a new sequence $O^{\prime}$ is generated by exchanging two aircraft sequences. Then, we calculate the difference of the objective function $\Delta F=F\left(O^{\prime}\right)-F(O)$. Here, $F(O)$ is the flight time of sequence $O$ and can get its value by the simulation model mentioned before. If $\Delta F<0$, the new sequence $O$ ' can reduce flight time; we replace $O$ with $O^{\prime}$, namely, $O=O^{\prime}$. Otherwise, the acceptable probability of $O^{\prime}$ is $\exp (-\Delta F / T)$. Comparing $\exp (-\Delta F / T)$ with a random number rand which belongs to $(0,1)$, if $\exp (-\Delta F / T)>$ rand, $O=O^{\prime}$; if $\exp (-\Delta F / T) \leq$ rand, we keep the sequence as same. Finally, if the number of iterations is satisfied, the current solution is output as the optimal solution to end the inner loop.

(3) Outer Loop. If $T<T_{e}$, the current solution can be regarded as the optimal aircraft sequence and iteration can be terminated. Otherwise, if $T=T \cdot \varphi$, go to the inner loop and solve for the optimal solution at temperature $T$.

\section{Case Study}

In this section, the TMA of Shanghai Hongqiao International Airport is taken as the case to verify the role of the PM procedure optimization model and sequencing model in improving operational benefits for arrival aircraft. Firstly, the operating environment in the TMA of Shanghai Hongqiao International Airport is introduced, including the border of TMA and temporal and spatial distribution of air traffic. Then, the design and optimization experiment of the PM procedure is performed. After that, the sequencing experiment of the arrival aircraft is carried out.

5.1. Operating Background. Shanghai Approach is one of the busiest TMA in China. Its horizontal range is shown in Figure 9, and its vertical range covers $6000 \mathrm{~m}$ and below.

Hongqiao International Airport is the main airport in Shanghai Approach. In 2019, it handled 272,928 flights and $45,637,882$ passengers, which makes it become one of China's major hubs and gateways to the outside world. It is a $4 \mathrm{E}$ civil international airport with a short distance parallel 


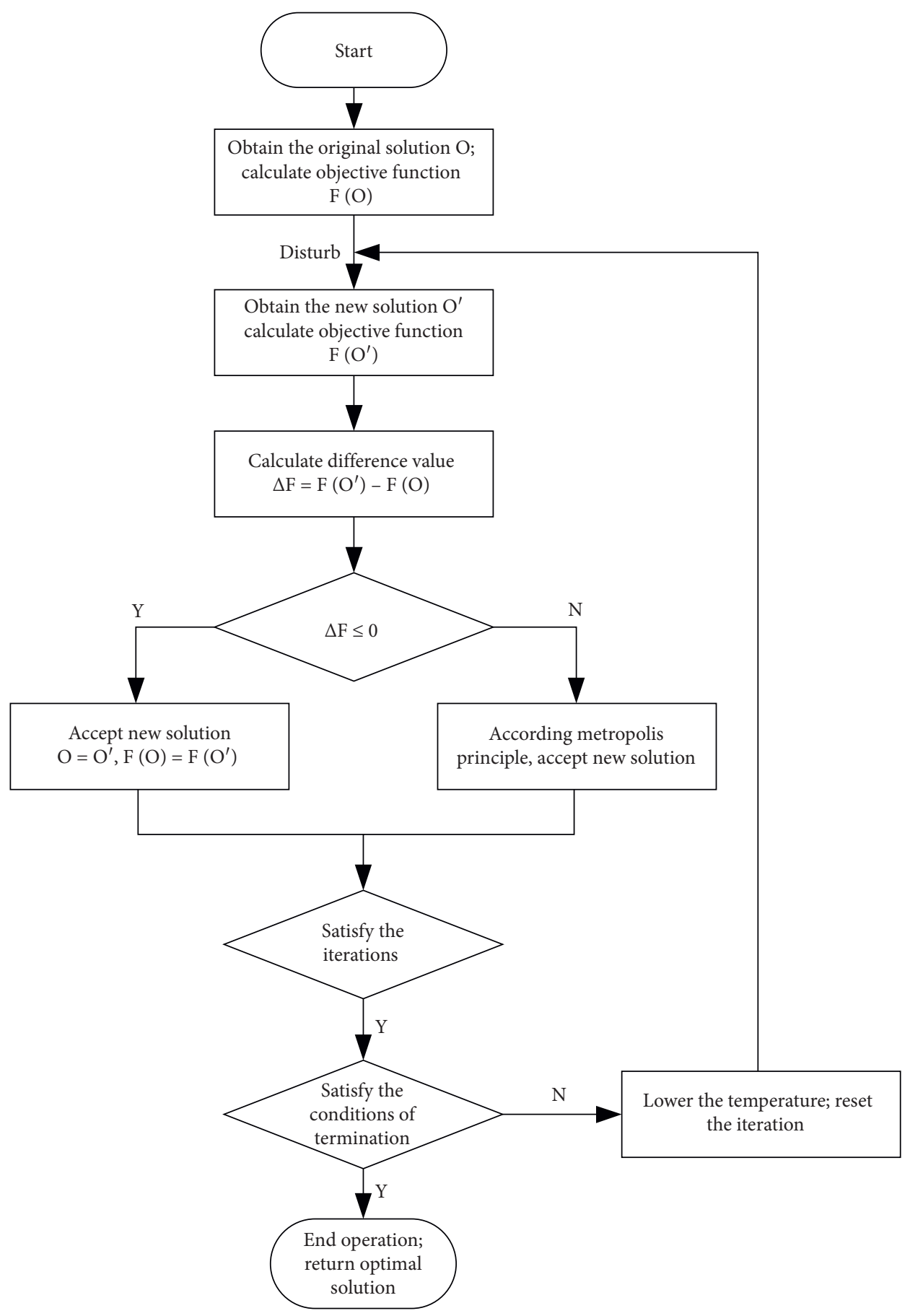

FIGURE 8: Flow diagram of simulated annealing (SA) algorithm.

runway of which operation mode is runway 36L-18R for take-off and landing and runway 36R-18L mainly for takeoff. We focused on the arrival traffic flow of runway 36L.

As shown in Figure 10, arrival traffic of runway 36L can enter the TMA through three entry points, including PUD in the east, AND in the south, and SASAN in the west.

Among them, 58\% of arrival aircraft enters TMA via the south point AND (AND-1F/42.34\%; AND-5F/16.51\%). Traffic from SASAN accounts for $39.23 \%$ and traffic from PUD accounts for only 1.91\%, as shown in Figure 11.
As for traffic flow data, we selected July 25, 2018, as a typical busy day of Hongqiao Airport as our flight data sample. The traffic flow from 00:00 to $23: 59$ on July 25, 2018, is shown in Figure 12.

5.2. Generation and Optimization of PM Procedure. According to the method mentioned in Section 2.1, combining the environment of Shanghai Hongqiao international airport, we placed the main structure of the PM procedure 


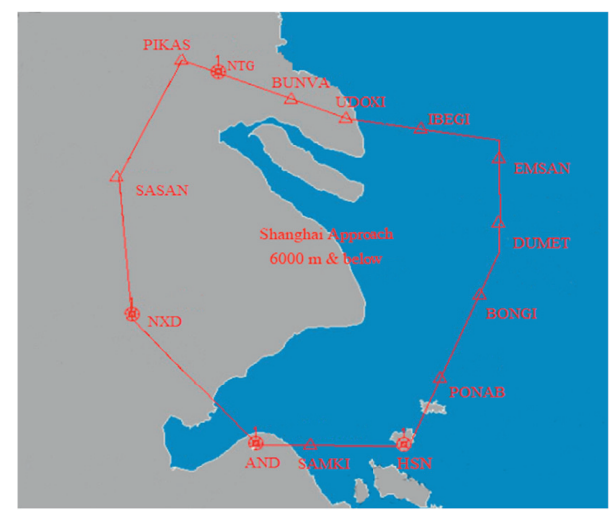

Figure 9: The border of Shanghai Approach.

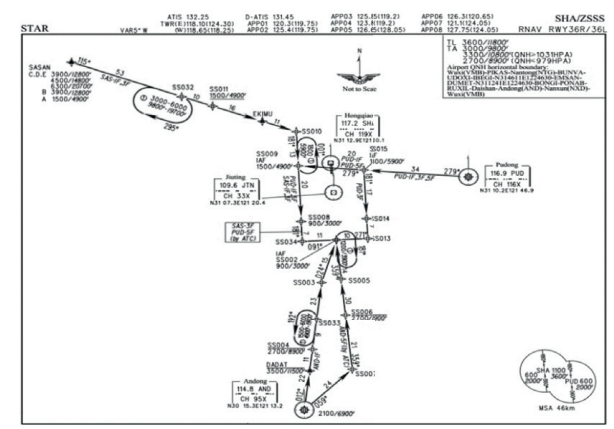

FIgURE 10: STAR of Shanghai Hongqiao International Airport.

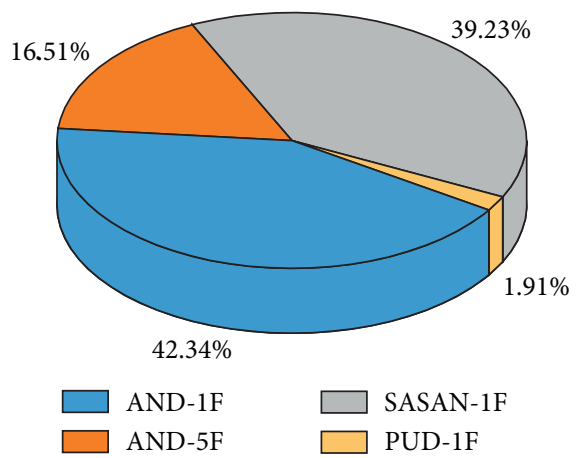

Figure 11: Temporal distribution of arrival flow.

on the south side of the airport. The fix JTN located on the extension of the runway centerline and $11 \mathrm{~nm}$ away from the threshold of runway is selected as the merge point. According to the direction of arrival traffic flow, we initially set the merge angle as $100^{\circ}$ and offset angle as $0^{\circ}$. The altitude of merge point is $900 \mathrm{~m}$, and the altitude of sequencing leg is $2700 \mathrm{~m}$.

Based on the initial PM procedure, the critical elements of PM procedure in Shanghai Hongqiao airport were substituted into the optimization model to verify the effectiveness of the optimization model. After calculation by GA, the fitness rapidly decreased from 0.95 to 0.75 within 200 generations and then declined slowly to 0.73 within 600 generations, and finally, the fitness tended to be stable after 700 generations, as shown in Figure 13.

After optimization, the value of merge angle equals $80^{\circ}$, the value of offset angle equals $-15^{\circ}$, the altitude of merge point is $1200 \mathrm{~m}$, and the altitude of sequencing leg is $3000 \mathrm{~m}$. The horizontal projection of the initial PM procedure and the optimized PM procedure on Google Earth is shown in Figure 14(a) and 14(b). It can be seen from the figure that the merge angle of the optimized PM procedure is significantly reduced, and the main body of the procedure is more anchored to the west.

5.3. Arrival Aircraft Sequencing. In order to further improve the operational benefits of arrival aircraft, flight data during the peak hour 13:00 13:59 were substituted into the sequencing model described in Section 4. For the same traffic flow in the initial PM procedure, the SA algorithm training process is shown in Figure 15. After sequencing, the overall flight time of the arrival aircraft was rapidly reduced from $890 \mathrm{~min}$ to $770 \mathrm{~min}$ within 100 generations, then reduced to $770 \mathrm{~min}$ within 500 generations, and became stable at 660 min after 700 generations.

After the aircraft sequencing, the landing sequence of the arrival aircraft changed. Take the fifth, sixth, and seventh aircraft as an example. After sequencing, the landing sequence has changed from 5-6-7 to 5-7-6. The fifth aircraft was the first to arrive following PUD-1F, the sixth aircraft was the second to arrive following AND-1F, and the seventh aircraft was the third to arrive following SASAN-1F. At this time, the position of each aircraft in the initial PM procedure is shown in Figure 16. The fifth aircraft reached the sequencing leg first and had no flight conflicts with the preceding and trailing aircraft, so it could fly directly to the merge point for the final approach.

Then, the sixth aircraft entered the sequencing leg. If it flies directly to the merge point, it will cause conflict with the fifth aircraft. Therefore, the sixth aircraft had to continue to fly along the sequencing leg until enough separation is established with the fifth aircraft, as shown in Figure 17. During this process, the seventh aircraft approached the sequencing leg.

When the seventh aircraft entered the sequencing leg, the sixth and seventh aircraft both met the safety separation with the fifth aircraft, but the two could not leave the sequencing leg simultaneously. According to the principle of First Come First Service (FCFS), the controller should choose to make the sixth aircraft turn and fly directly, but the sequencing model calculation results showed that the seventh aircraft turned first for the final approach which can make the average flight time of each arrival aircraft shorter, which is more conducive to the operational benefits of the whole arrival traffic. Therefore, as shown in Figure 18, the landing sequence has changed from 5-6-7 to 5-7-6.

By analogy, during the peak hour from 13:00 to $13: 59$, the corresponding landing sequence and actual landing time of 31 arrival aircraft under different situations are shown in Table 4. On one hand, the effectiveness of the PM procedure optimization model can be verified by comparing the 


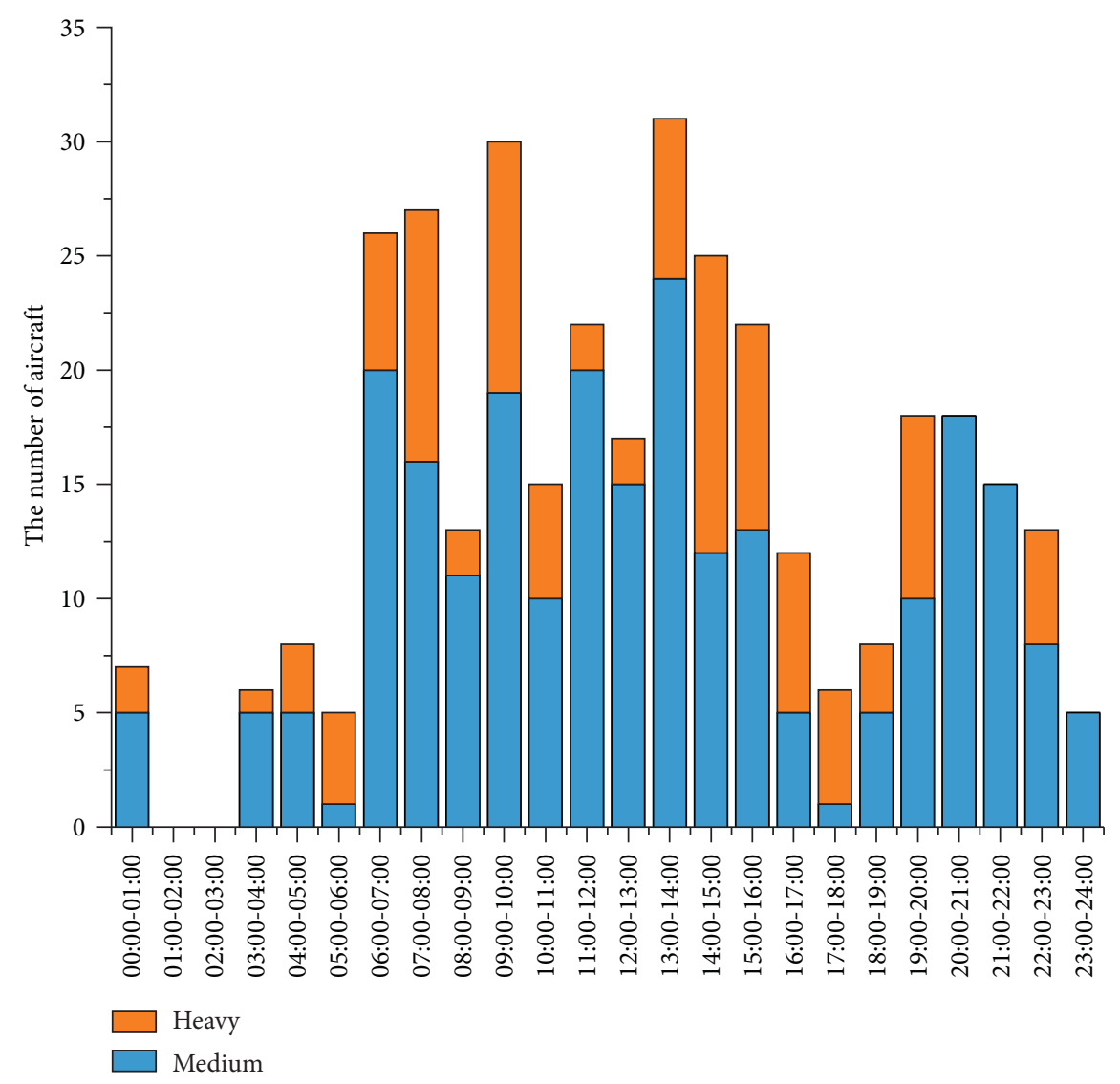

FIgURE 12: Distribution of aircraft types.

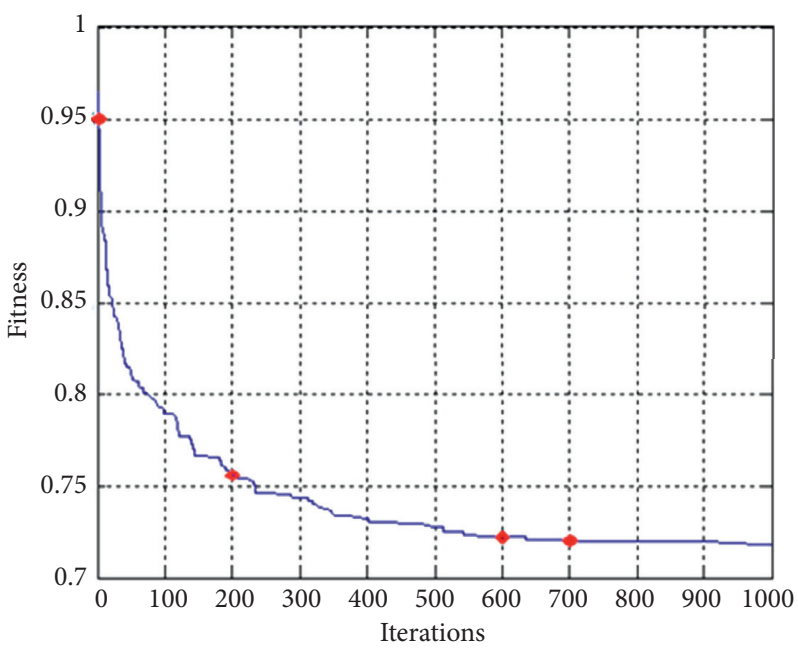

Figure 13: Genetic Algorithm (GA) training process.

landing time of arrival aircraft in different procedures while adopting the same sequence. On the other hand, the effectiveness of the sequencing model can be verified by comparing the landing time of arrival aircraft in the same procedure while adopting different sequences.

In order to make the results more obvious, we selected the 1th to 21th arrival aircraft and plotted their landing times, as shown in Figure 19.
From the information in Figure 19, we can draw the following conclusions:

(1) For the same flow of arrival aircraft adopting the FCFS landing sequence, the landing time of aircraft in the initial PM procedure is significantly earlier than the landing time in the STAR. For the first five aircraft, the advantage of the PM procedure is not 


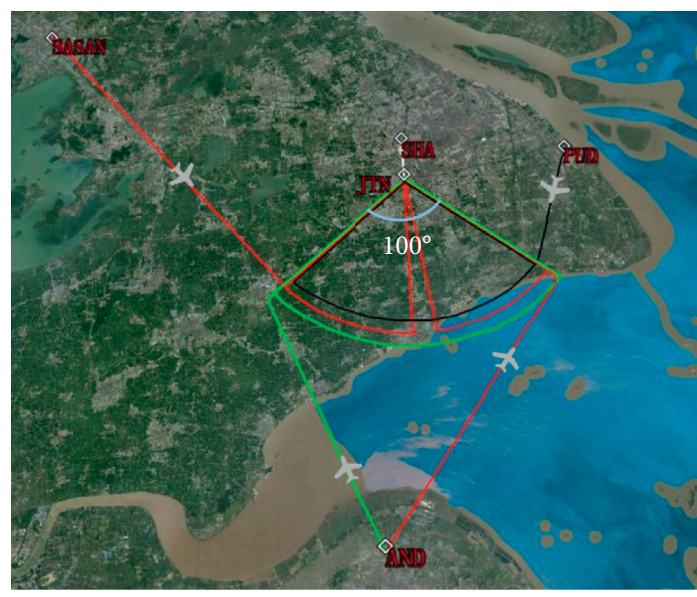

- AND-1A

- PUD-1A

SASAN-1A AND-5A

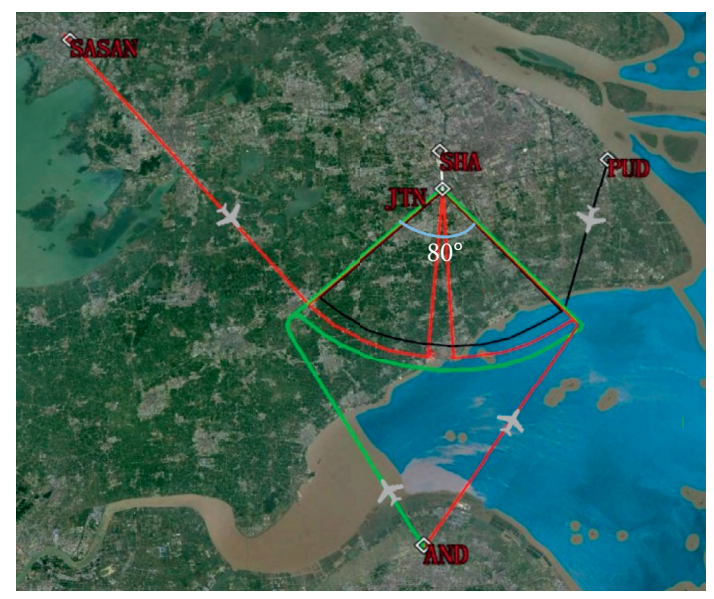

- AND-1A

- PUD-1A

SASAN-1A

AND-5A

(a)

(b)

FIgUre 14: Comparison of two different PM procedures on Google Earth. (a) Initial PM procedure. (b) Optimized PM procedure.

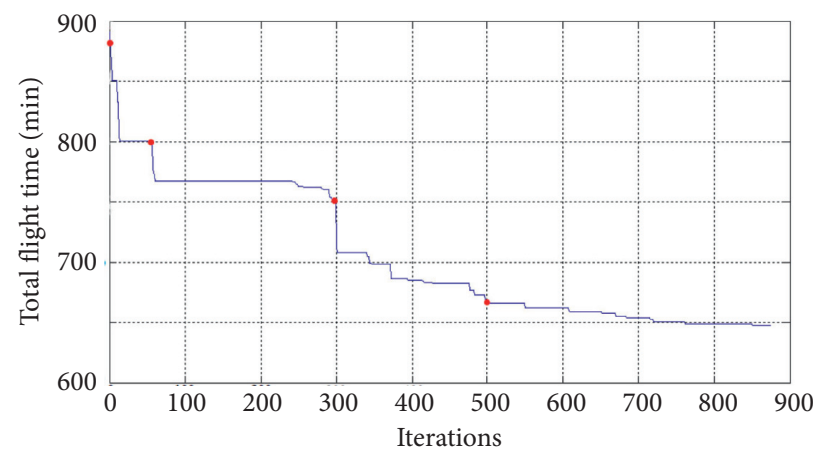

FIGURE 15: SA algorithm training process.

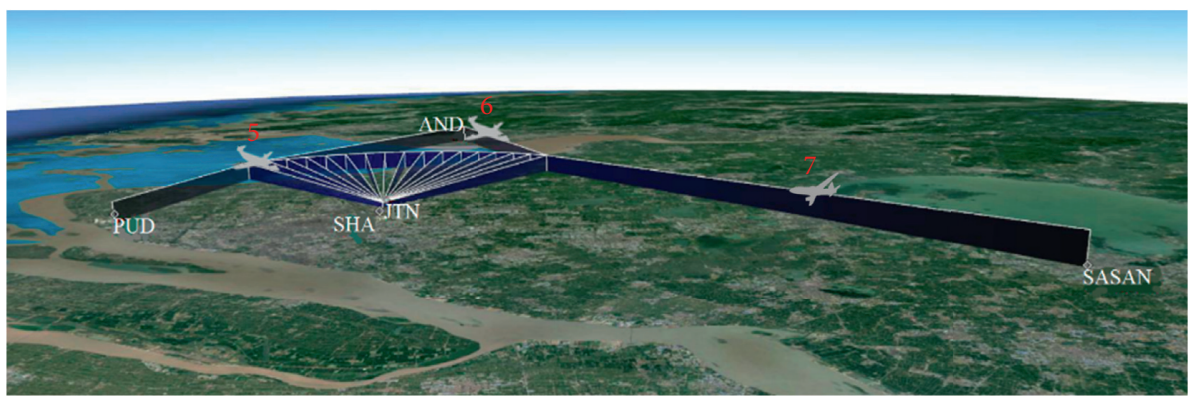

FIGURE 16: Aircraft arriving from different directions.

obvious, because the number of arrival aircraft in the procedure is small at the beginning of simulation, and the aircraft did not need to wait. As time accumulated, the number of aircraft in the procedure gradually increased, and PM procedure showed great advantages to accommodate more aircraft and significantly reduce the flight time of aircraft.
(2) For the same flow of arrival aircraft in the initial PM procedure, the landing time of aircraft after sequencing is obviously earlier than the landing time of FCFS. In addition, the point which represents the sequence of each aircraft in the figure has not exchanged too much. Only one aircraft has exchanged for two positions, from the 12th to the 14th. Most of 


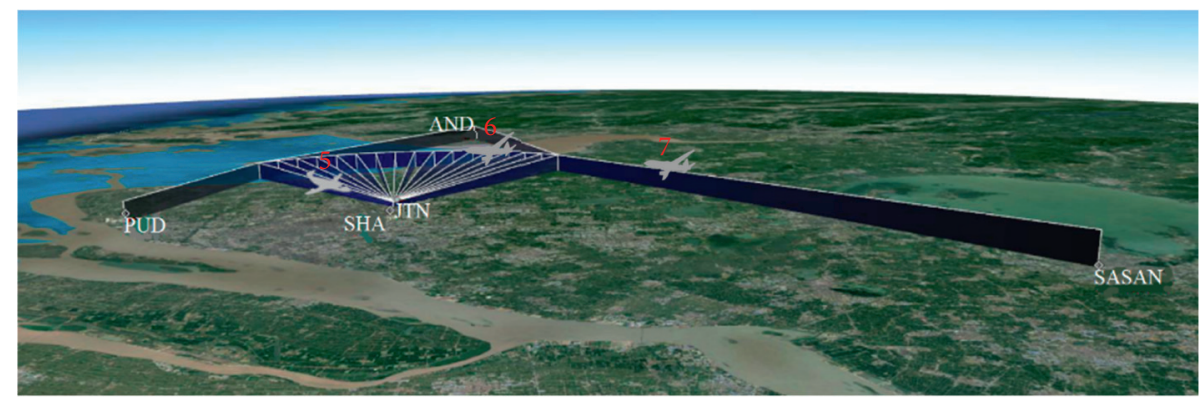

FIgURE 17: Aircraft flying along the sequencing leg.

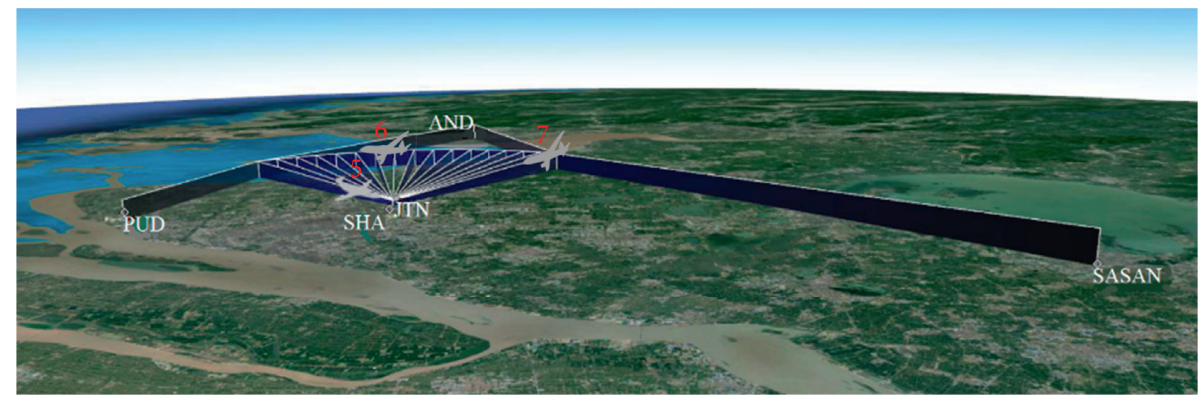

Figure 18: Change of aircraft landing sequence.

TABLE 4: Sequencing results of aircraft under different conditions.

\begin{tabular}{|c|c|c|c|c|c|c|c|}
\hline \multicolumn{2}{|c|}{ STAR-FCFS } & \multicolumn{2}{|c|}{ Initial PM procedure FCFS } & \multicolumn{2}{|c|}{$\begin{array}{l}\text { Initial PM procedure after } \\
\text { sequencing }\end{array}$} & \multicolumn{2}{|c|}{$\begin{array}{l}\text { Optimized PM procedure after } \\
\text { sequencing }\end{array}$} \\
\hline Landing sequence & Landing time & Landing sequence & Landing time & Landing sequence & Landing time & Landing sequence & Landing time \\
\hline 1 & $13: 15: 02$ & 1 & $13: 15: 18$ & 1 & $13: 15: 18$ & 1 & $13: 14: 31$ \\
\hline 2 & $13: 17: 13$ & 2 & $13: 17: 15$ & 2 & $13: 17: 15$ & 2 & $13: 16: 23$ \\
\hline 3 & $13: 19: 22$ & 3 & 13:19:15 & 3 & 13:19:15 & 3 & $13: 18: 34$ \\
\hline 4 & $13: 22: 57$ & 4 & $13: 22: 31$ & 4 & $13: 22: 31$ & 4 & $13: 20: 51$ \\
\hline 5 & $13: 25: 10$ & 5 & $13: 24: 33$ & 5 & $13: 24: 33$ & 5 & $13: 23: 17$ \\
\hline 6 & $13: 28: 32$ & 6 & $13: 27: 49$ & 7 & $13: 25: 47$ & 7 & $13: 24: 42$ \\
\hline 7 & $13: 30: 41$ & 7 & $13: 29: 25$ & 6 & $13: 27: 56$ & 6 & $13: 26: 38$ \\
\hline 8 & $13: 32: 38$ & 8 & $13: 31: 13$ & 9 & $13: 29: 34$ & 9 & $13: 28: 21$ \\
\hline 9 & $13: 35: 59$ & 9 & $13: 34: 21$ & 8 & $13: 30: 52$ & 8 & $13: 29: 53$ \\
\hline 10 & $13: 37: 21$ & 10 & $13: 35: 12$ & 11 & $13: 32: 13$ & 10 & $13: 31: 26$ \\
\hline 11 & $13: 40: 24$ & 11 & $13: 37: 35$ & 10 & $13: 33: 45$ & 11 & $13: 32: 43$ \\
\hline 12 & $13: 42: 31$ & 12 & $13: 39: 25$ & 13 & $13: 35: 23$ & 13 & $13: 34: 17$ \\
\hline 13 & $13: 44: 52$ & 13 & $13: 41: 15$ & 14 & $13: 36: 47$ & 14 & $13: 35: 35$ \\
\hline 14 & $13: 46: 25$ & 14 & $13: 42: 36$ & 12 & $13: 38: 52$ & 12 & $13: 37: 16$ \\
\hline 15 & $13: 47: 33$ & 15 & $13: 43: 12$ & 15 & $13: 40: 03$ & 15 & 13:39:04 \\
\hline 16 & $13: 49: 27$ & 16 & $13: 45.33$ & 17 & $13: 41: 35$ & 16 & $13: 40: 28$ \\
\hline 17 & $13: 50: 48$ & 17 & $13: 46: 54$ & 16 & $13: 43: 14$ & 17 & $13: 42: 19$ \\
\hline 18 & $13: 52: 19$ & 18 & $13: 48: 12$ & 18 & $13: 44: 57$ & 18 & $13: 43: 38$ \\
\hline 19 & $13: 53: 44$ & 19 & $13: 49: 37$ & 19 & $13: 46: 38$ & 19 & $13: 45: 06$ \\
\hline 20 & $13: 55: 06$ & 20 & $13: 51: 23$ & 21 & $13: 48: 21$ & 21 & $13: 47: 27$ \\
\hline 21 & $13: 56: 37$ & 21 & $13: 53: 15$ & 20 & $13: 49: 36$ & 20 & $13: 48: 52$ \\
\hline 22 & $13: 58: 51$ & 22 & $13: 54: 36$ & 23 & $13: 51: 29$ & 23 & $13: 50: 24$ \\
\hline 23 & $14: 00: 02$ & 23 & $13: 55: 48$ & 22 & $13: 52: 33$ & 22 & $13: 51: 39$ \\
\hline 24 & $14: 01: 37$ & 24 & $13: 57: 21$ & 24 & $13: 53: 48$ & 24 & $13: 53: 17$ \\
\hline 25 & $14: 02: 56$ & 25 & $13: 58: 52$ & 26 & $13: 55: 16$ & 26 & $13: 54: 29$ \\
\hline 26 & $14: 04: 03$ & 26 & $13: 59: 58$ & 25 & $13: 56: 29$ & 25 & $13: 56: 12$ \\
\hline 27 & $14: 06: 24$ & 27 & $14: 01: 13$ & 28 & $13: 58: 02$ & 28 & $13: 57: 36$ \\
\hline 28 & 14:08:07 & 28 & $14: 02: 27$ & 29 & $13: 59: 34$ & 27 & 13:58:51 \\
\hline 29 & $14: 09: 21$ & 29 & $14: 04: 22$ & 30 & $14: 01: 21$ & 30 & $14: 00: 26$ \\
\hline 30 & $14: 11: 15$ & 30 & $14: 06: 46$ & 27 & $14: 02: 34$ & 29 & 14:01:39 \\
\hline 31 & $14: 13: 36$ & 31 & $14: 07: 33$ & 31 & $14: 03: 57$ & 31 & $14: 02: 45$ \\
\hline
\end{tabular}




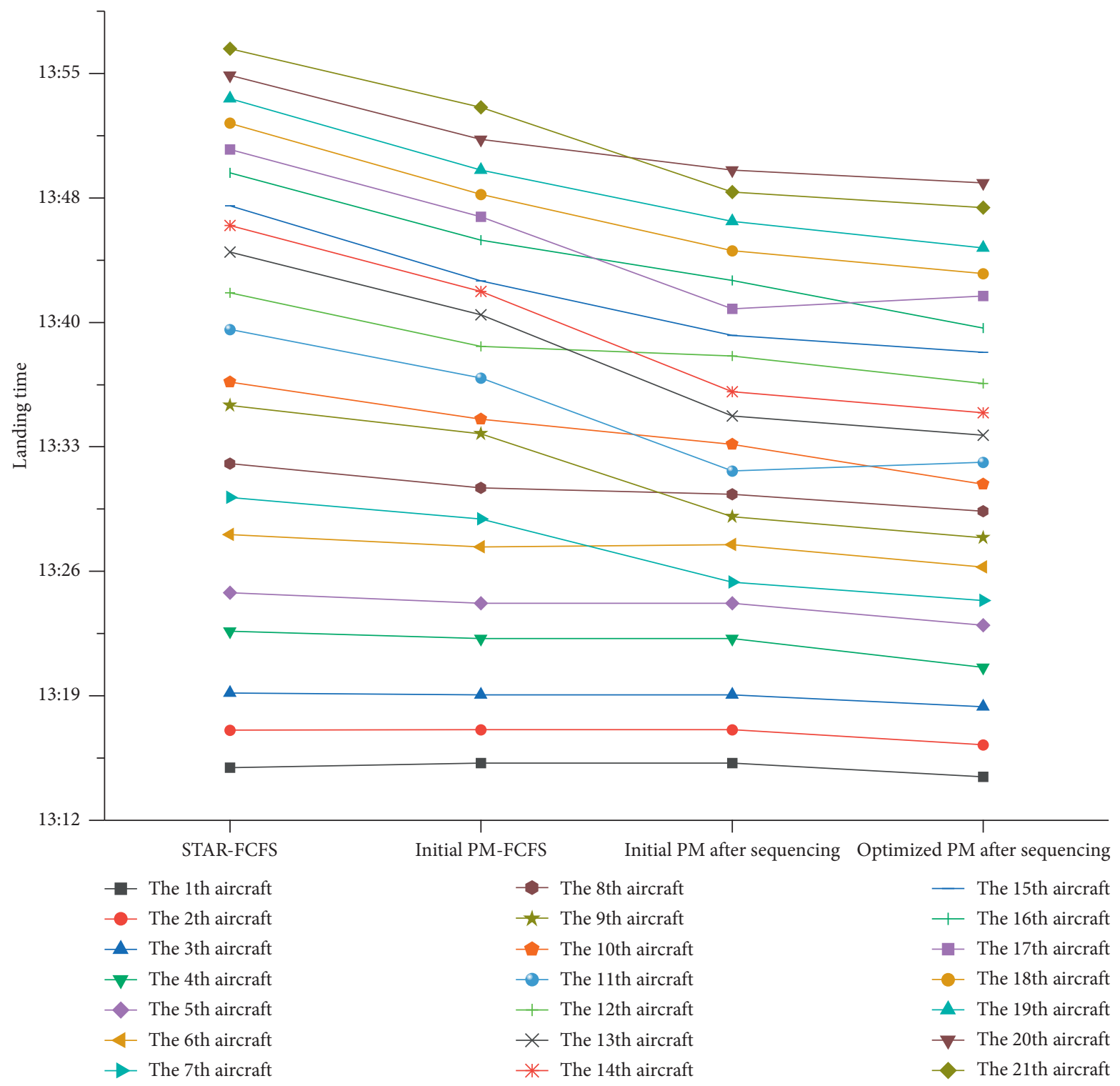

Figure 19: Actual landing time of aircraft under different conditions.

the aircraft positions remain still or only one position is exchanged, but the actual landing time is greatly advanced, thus improving the operational benefits of arrival aircraft without excessively increasing the workload of the controller.

(3) Combining the PM procedure optimization model with the sequencing model can dramatically advance the landing time of arrival aircraft. The slopes of three segments of each line in Figure 19 are all less than 0 , indicating that the PM procedure optimization model and the sequencing model can shorten the flight time, but the absolute value of the slope of the second segment is significantly greater than that of the third segment, which indicates that the sequencing model seems to have a more obvious effect on the landing time of arrival aircraft than the PM procedure optimization model.
5.4. Operational Benefits Comparison. On the premise of safety, improving the operational benefits of arrival aircraft is an important goal of civil aviation development. Operational benefits include economic benefits and environmental benefits. Based on the PM procedure optimization experiment and the sequencing experiment, we calculated average flight time and average fuel consumption representing the economic benefits, average pollutant emissions, and noise impact range representing the environmental benefits of the arrival aircraft under five conditions. The results are shown in Figure 20.

According to the results above, we can get the following conclusions:

(1) For the arrival procedure, from the STAR to the initial PM procedure to the optimized PM procedure, the operational benefits are gradually improved. In particular, the two indicators of average 


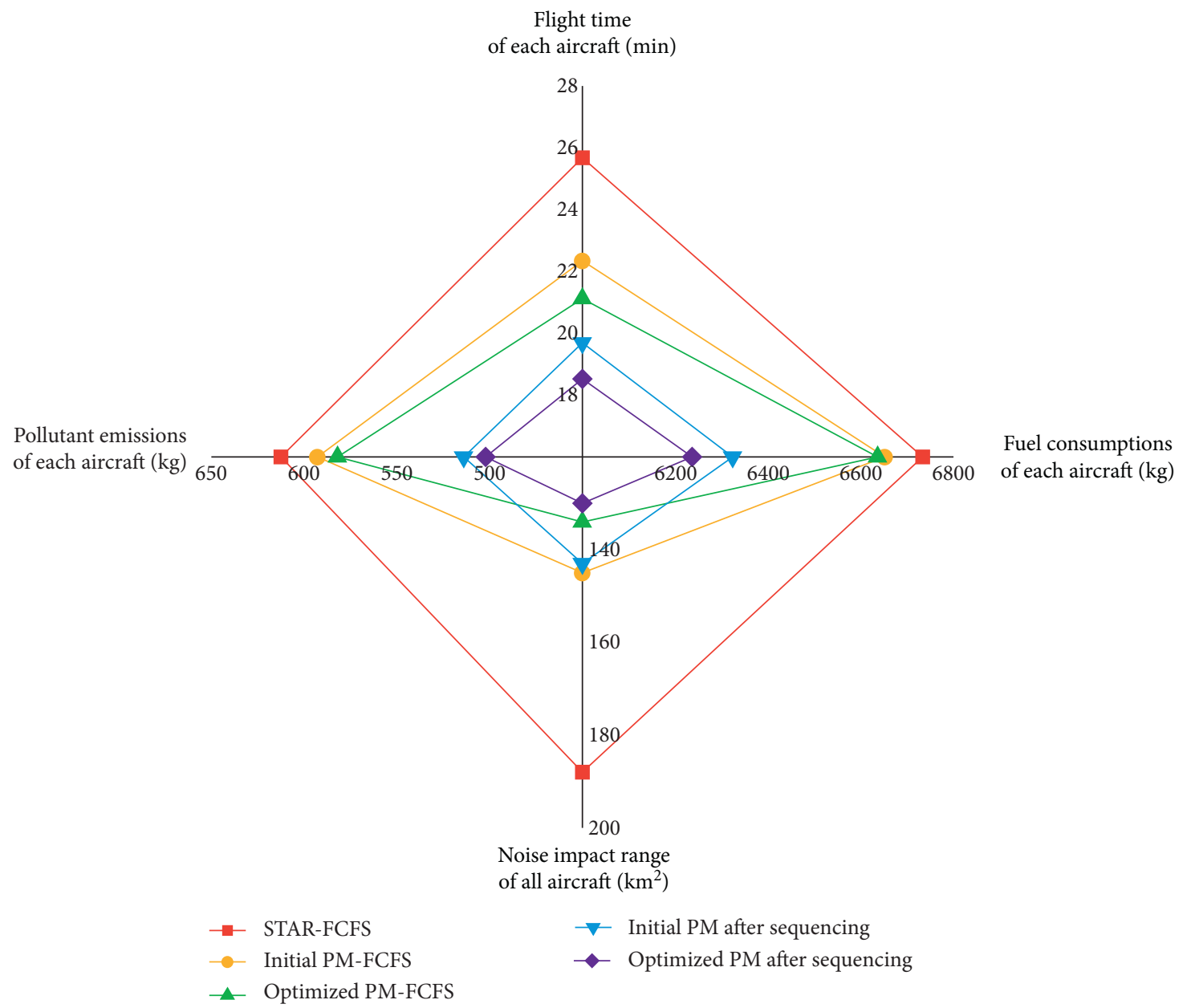

Figure 20: Comparison of operational benefits of arrival aircraft.

flight time and noise impact range have been significantly improved. Compared with the STAR, the initial PM procedure can reduce the average flight time by 3.34 minutes, a reduction of $13 \%$, and the noise impact range by $43 \mathrm{~km}^{2}$, a reduction of $22.87 \%$. Compared with the initial PM procedure, the optimized PM procedure can reduce the average flight time by 1.22 minutes, a decrease of $5.46 \%$, and the noise influence range by $11 \mathrm{~km}^{2}$, a reduction of $7.59 \%$.

(2) On the basis that the application of PM procedure can improve flight operational benefits, comparing FCFS and PM sequencing on the operational benefits of arrival aircraft, it can be found that the landing sequence generated by the PM sequencing model is significantly better than FCFS. The two indicators of average flight time and pollutant emissions have been significantly improved. The PM sequencing model applied to the initial PM procedure and the optimized PM procedure can reduce the average flight time by $2.65 \mathrm{~min}$ and $2.59 \mathrm{~min}, 11.86 \%$ and $12.26 \%$, and reduce the average pollutant emissions by $78.86 \mathrm{~kg}$ and $79.73 \mathrm{~kg}$, a reduction of $13.30 \%$ and $13.74 \%$.
(3) In general, the application of the PM procedure optimization model and sequencing model can improve the operational benefits of the arrival aircraft. However, the PM procedure optimization model improves the noise impact range more significantly, while the sequencing model improves the average pollutant emissions more significantly. Therefore, the combination of the two can maximize the operational benefits of arrival aircraft.

Based on the noise calculation results, we drew the noise surface diagram of the same arrival flow in STAR, initial PM procedure, and optimized PM procedure, as shown in Figure 21(a)-21(c). Through Figure 21, we can obviously note that the noise generated by the same arrival flow in the optimized PM procedure has the least impact range.

The pollutants in Figure 20 include $\mathrm{CO}, \mathrm{HC}$, and $\mathrm{NO}_{x}$ which are the main pollutants produced by arrival aircraft in TMA. The specific emissions of the three pollutants can be seen in Figure 22.

Compared with STAR-FCFS, the three pollutants under the other four conditions all reduced, especially under the condition of optimized procedure after sequencing. The emissions $\mathrm{CO}$ and $\mathrm{HC}$ under the condition of optimized 


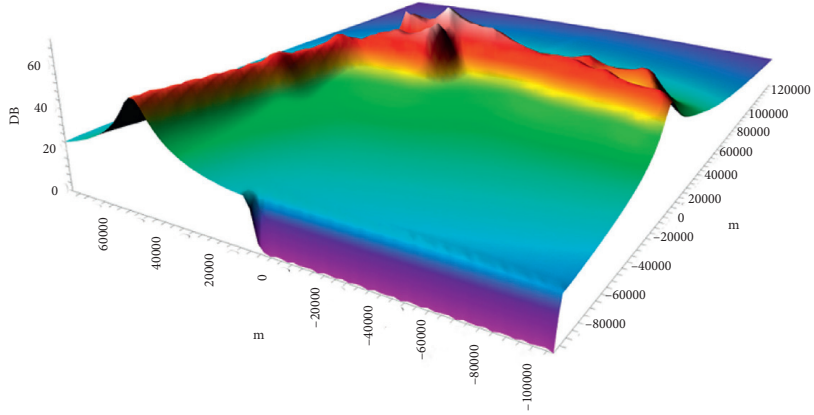

(a)

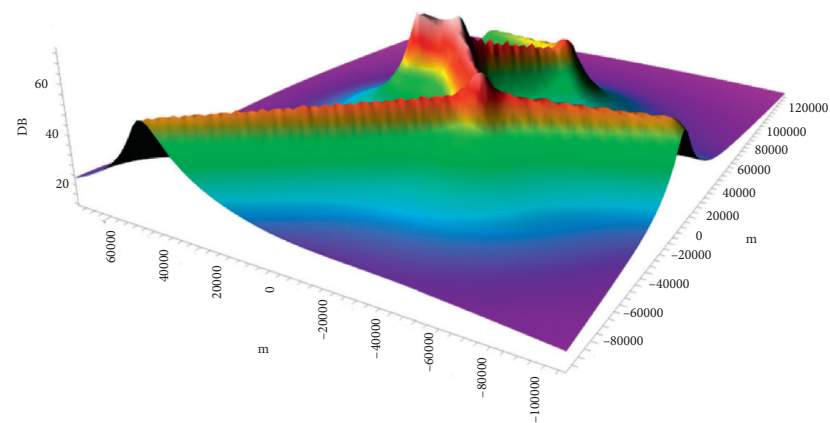

(b)

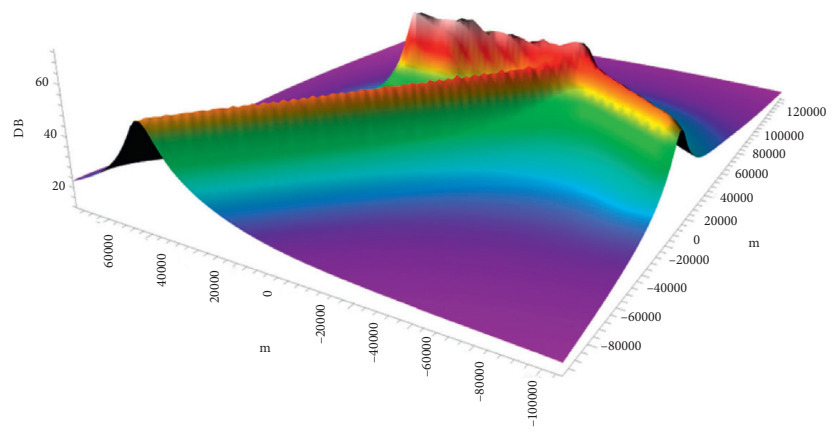

(c)

FIgURE 21: Noise surface diagram of the same arrival flow in three different flight procedures. (a) STAR. (b) Initial PM procedure. (c) Optimized PM procedure.

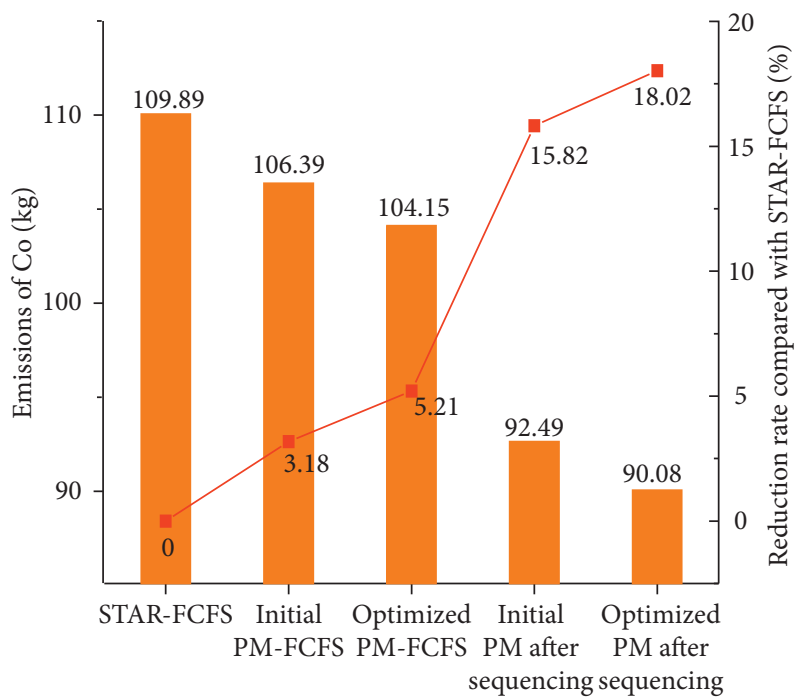

Emissions of $\mathrm{Co}$

- - - Reduction rate compared with STAR-FCFS

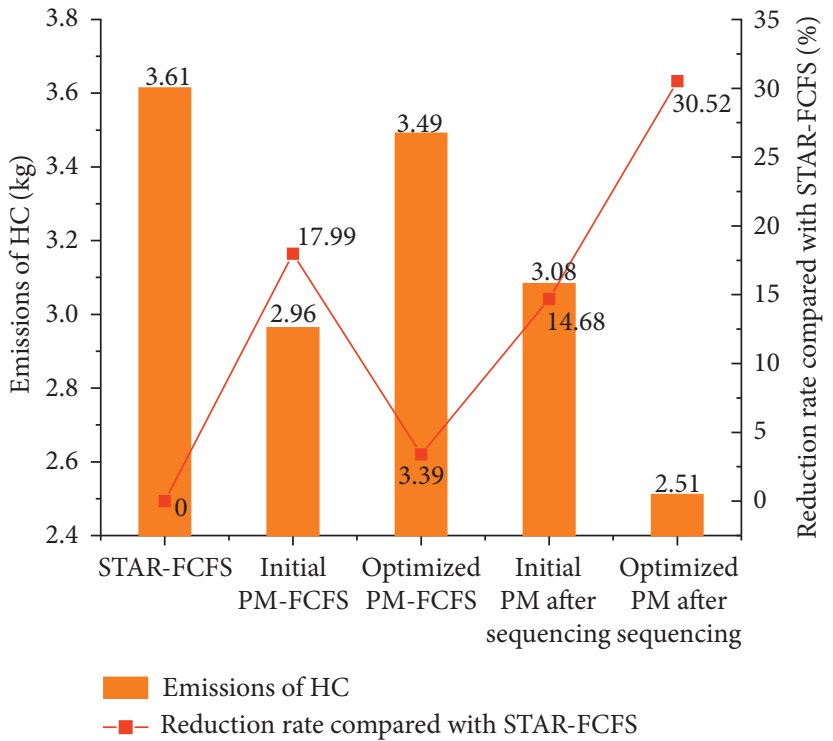

(b)

Figure 22: Continued. 


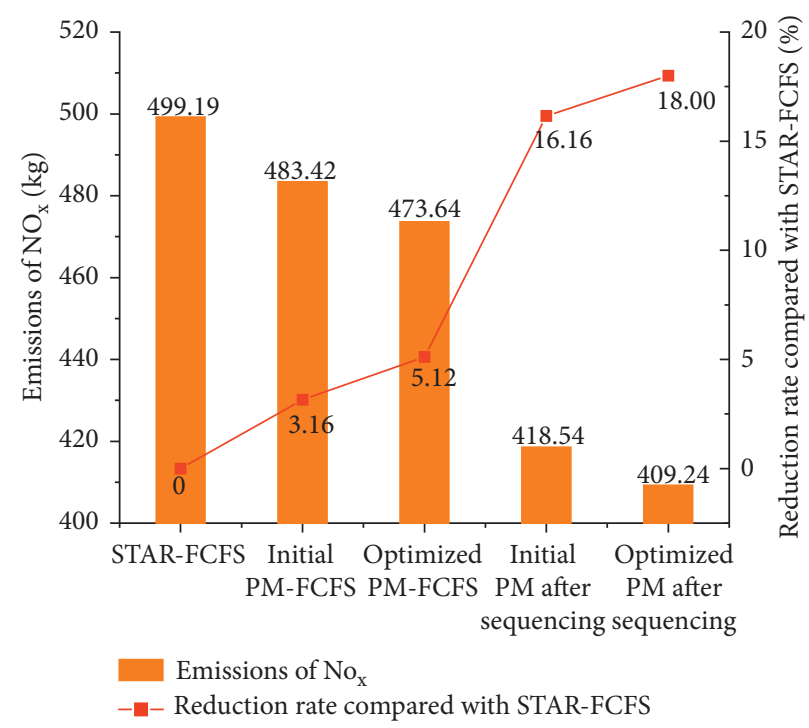

(c)

Figure 22: The emissions of three pollutants. (a) CO. (b) HC. (c) $\mathrm{NO}_{x}$.

procedure after sequencing reduced by $18 \%$ compared to STAR-FCFS, as for the emissions of HC reduced by $30 \%$. Among the three pollutants, $\mathrm{NO}_{x}$ emissions are the largest, and the average emissions of $\mathrm{NO}_{x}$ for each aircraft can be reduced by $90 \mathrm{~kg}$.

\section{Conclusion}

This paper focuses on how to apply the PM procedure to maximize the operational benefits of arrival aircraft. The method that we use can provide the reference for the airports which require new flight procedures to address the imbalance between excessive arrival traffic and relatively low operational benefits. Firstly, we introduce the framework of the PM procedure, including its structure and the corresponding aircraft operation mode, which improves the current frequent congestion in TMA, thus promoting the transition from STAR to the PM procedure. Then the optimal approach route can be provided for each arrival aircraft through the optimization model of the PM procedure. Furthermore, the optimal landing sequence of arrival traffic flow can be obtained through the aircraft sequencing model, thus dramatically improving the operational benefits, not only economic benefits but also environmental benefits. Finally, the TMA of Shanghai Approach is taken as the case to verify the effectiveness of the two models. The experimental results show that the combination of the two can dramatically improve the operational benefits of arrival aircraft, thereby meeting the sustainable development requirements of TMA.

Except the methods used in the paper, some of the most representative computational intelligence algorithms also can be used to solve the problems in this paper, like Monarch Butterfly Optimization (MBO), Earthworm Optimization Algorithm (EWA), Elephant Herding Optimization (EHO), Moth Search (MS) Algorithm, Slime Mould Algorithm
(SMA), and Harris Hawks Optimization (HHO). In the next step of our research, we will compare these algorithms with the methods proposed in this paper. Besides, with traffic flow surges, more and more cities are building multiple airports. The application of PM procedure in TMA with multiple airports can be further studied.

\section{Data Availability}

The data used to support the findings of this study are available from the corresponding author upon request.

\section{Conflicts of Interest}

The authors declare no conflicts of interest.

\section{Acknowledgments}

This research was funded by the National Natural Science Foundation of China, Grant no. 61671237. This research was also funded by the Foundation of the Graduate Innovation Center in Nanjing University of Aeronautics and Astronautics, Grant no. kfjj20200735.

\section{References}

[1] CAAC, 2019 Statistica l Bulletin of Civil Aviation Industry Development, China Civil Aviation Administration, Beijing, China, 2020.

[2] L. Boursier, F. Bruno, E. Hoffman, A. Trzmiel, F. Vergne, and K. Zeghal, "Merging arrival flows without heading instructions," in Proceedings of the Paper presented at the 7th USA/ Europe Air Traffic Management R\&D Seminar, Barcelona, Spain, July 2007.

[3] E. Aydogan and C. Cetekt, "Point merge concept for en route air traffic flow management," Journal of Aircraft, vol. 55, no. 6, pp. 1-13, 2018. 
[4] Eun et al., "Research and development trend of trajectorybased operations in air traffic management," Current Industrial and Technological Trends in Aerospace, vol. 12, pp. 192-199, 2014.

[5] A. Errico and V. D. Vito, "Study of point merge technique for efficient continuous descent operations in TMA," IFACPapersOnLine, vol. 51, no. 9, pp. 193-199, 2018.

[6] D. Ivanescu, C. Shaw, C. Tamvaclis, and T. Kettunen, "Models of air traffic merging techniques: evaluating performance of point merge," in Proceedings of the Paper presented at the 9th AIAA Aviation Technology, Integration, and Operations Conference (ATIO) and Aircraft Noise and Emissions Reduction Symposium (ANERS), Hilton Head, SC, USA, September 2009.

[7] B. Favennec, E. Hoffman, A. Trzmiel, F. Vergne, and K. Zeghal, "The point merge arrival flow integration technique: towards more complex environments and advanced continuous descent," in Proceedings of the Paper presented at the 9th AIAA Aviation Technology, Integration, and Operations Conference (ATIO) and Aircraft Noise and Emissions Reduction Symposium (ANERS), Hilton Head, SC, USA, September 2009.

[8] Ö. Sahin Meric and O. Usanmaz, "A new standard instrument arrival: the point merge system," Aircraft Engineering and Aerospace Technology, vol. 85, no. 2, pp. 136-143, 2013.

[9] C. MianBo, X.-L. Zhao, L. I. Peng-Cheng, and Bi-Yu Lei, "Research on point merge approach procedure design in terminal area," Aeronautical Computing Technique, vol. 46, no. 2, pp. 14-18, 2016.

[10] Z. Xiangling and B. Lei, "Research on method for assessing risk in ground approach under point merging procedure," China Safety Ence Journal, 2017.

[11] Qi Yannan, X. Wang, and C. Chen, "Research on arrival integration method for point merge system in tactical operation," in Proceedings of the Paper presented at the International Conference on Combinatorial Optimization and Applications, Shanghai, China, December 2017.

[12] O. Sahin, O. Usanmaz, and E. T. Turgut, "An assessment of flight efficiency based on the point merge system at metroplex airports," Aircraft Engineering and Aerospace Technology, vol. 90, no. 1, pp. 1-10, 2018.

[13] L. Man, "An agent-based approach to automated merge $4 \mathrm{~d}$ arrival trajectories in busy terminal maneuvering area," Procedia Engineering, vol. 99, pp. 233-243, 2015.

[14] L. Man, D. Delahaye, M. Sbihi, and Ji Ma, "Multi-layer point merge system for dynamically controlling arrivals on parallel runways," in Proceedings of the Paper presented at the 2016 IEEE/AIAA 35th Digital Avionics Systems Conference (DASC), Sacramento, CA, USA, September 2016.

[15] L. Man, D. Delahaye, and P. Maréchal, "Integrated sequencing and merging aircraft to parallel runways with automated conflict resolution and advanced avionics capabilities," Transportation Research Part C: Emerging Technologies, vol. 85, pp. 268-291, 2017.

[16] T. Yong, D. Xing, L. Wan, and B. Ye, "Study on the optimization method of point merge procedure based on benefit in the terminal area," Mathematical Problems in Engineering, p. 2020, 2020.

[17] L. Man, DELAHAYE Daniel, and MARECHAL Pierre, "Potential operational benefits of multi-layer point merge system on dense tma operation," in Proceedings of the Paper presented at the 7th International Conference on Research in Air Transportation (ICRAT), Philadelphia, PA, USA, June 2016.
[18] C. Xiangan, Research, Optimization, Seqencing of a Point Merge System in Tma Approach, Civil Aviation University of China, Tianjin, China, 2016.

[19] Jianzhong, C. Wang and B. C. Zhang, 4d trajectory planning method for arrivals based on merging point Approach," Science Technology \& Engineering, 2017.

[20] Y. Hong, B. Choi, S. Lee, K. Lee, and Y. Kim, "Optimal and practical aircraft sequencing and scheduling for point merge system," IFAC-PapersOnLine, vol. 50, no. 1, pp. 14644-14649, 2017.

[21] Y. Hong, B. Choi, K. Lee, and Y. Kim, "Dynamic robust sequencing and scheduling under uncertainty for the point merge system in terminal airspace," IEEE Transactions on Intelligent Transportation Systems, vol. 19, no. 9, pp. 29332943, 2017.

[22] Y. Hong, S. Lee, K. Lee, and Y. Kim, "Optimal scheduling algorithm for air traffic point merge system using milp," in Advances in Aerospace Guidance, Navigation and Control, pp. 407-420, Springer, Cham, Switzerland, 2018.

[23] S. Lee, Y. Hong, and Y. Kim, "Optimal scheduling algorithm in point merge system including holding pattern based on mixed-integer linear programming," Proceedings of the Institution of Mechanical Engineers, Part G: Journal of Aerospace Engineering, vol. 234, no. 10, pp. 1638-1647, 2020.

[24] J. Zhang, P. Zhao, C. Yang, and R. Hu, "A new meta-heuristic approach for aircraft landing problem [J]," Transactions of Nanjing University of Aeronautics and Astronautics, vol. 37, no. 02, pp. 197-208, 2020.

[25] K. D. Bilimoria, B. Sridhar, S. R. Grabbe, G. B. Chatterji, and K. S. Sheth, "Facet: future ATM concepts evaluation tool," Air Traffic Control Quarterly, vol. 9, no. 1, pp. 1-20, 2001.

[26] D G. Hull, Fundamentals of Airplane Flight Mechanics, Springer, Berlin, Germany, 2007.

[27] P. Brooker, "Air traffic control separation minima: part 2-transition to a trajectory-based system," Journal of Navigation, vol. 64, no. 4, p. 673, 2011.

[28] ICAO, Doc 4444, Air Traffic Management-Procedures for Air Navigation Services (Pans-Atm) Ed 16, ICAO, Montreal, Canada, 2009.

[29] A. Nikoleris and H. Erzberger, "Autonomous system for air traffic control in terminal airspace," in Paper presented at the 14th AIAA Aviation Technology, Integration, and Operations Conference, Atlanta, GA, USA, June 2014.

[30] Y. Tian, L. Wan, K. Han, and B. Ye, "Optimization of terminal airspace operation with environmental considerations," Transportation Research Part D: Transport and Environment, vol. 63, pp. 872-889, 2018.

[31] A. T. Ernst, C. Oğuz, G. Singh, and G. Taherkhani, "Mathematical models for the Berth allocation problem in dry bulk terminals," Journal of Scheduling, vol. 20, no. 5, pp. 459-473, 2017.

[32] J. Q. Li, Y. Q. Han, P. Y. Duan, Y. Y. Han, B. Niu et al., "Metaheuristic algorithm for solving vehicle routing problems with time windows and synchronized visit constraints in prefabricated systems," Journal of Cleaner Production, vol. 250, Article ID 119464, 2020. 